\title{
MEMBERS OF THE SOCIETY
}

\author{
October, 1928
}

(Life members are designated by $\dagger$; nominees of sustaining members by $\S$. )

§Аввотт, Eleanor A. John Hancock Mutual Life Insurance Co., 197 Clarendon St., Boston, Mass.

Ackermann-Teubner, Dr. B. G. A. Poststr. 3, Leipzig, Germany.

Adams, Asso. Prof. C. R. Brown Univ., Providence, R. I. 260 Doyle Ave.

Adams, Prof. E. P. Princeton Univ., Princeton, N. J. 245 Nassau St.

ADAMS, Dr. O. S. U. S. Coast and Geodetic Survey, Washington, D. C. 727 Massachusetts Ave, $N$ E.

Adams, Dr. RACHEL B. '(Mrs. C. R.). 260 Doyle Ave., Providence, R. I.

§ADkisson, Asst. Prof. V. W. Univ. of Arkansas, Fayetteville, Ark.

Adshead, Asst. Prof. J. G. Dalhousie Univ., Halifax, Nova Scotia.

AgARd, Asst. Prof. H. L. Williams Coll., Williamstown, Mass.

Agnew, Dr. R. P. National Research Fellow. Poland, Ohio.

Akers, Prof. O. P. Allegheny Coll, Meadeville, Pa. 360 N. Main St.

AlberT, Dr. A. A. Inst., Columbia University, New York, N. Y. Hamilton Hall.

Albert, Prof. O. W. Univ. of Redlands, Redlands, Calif. 629 Buena Vista St.

ALDEN, H. H. 640 Green Ave. S. W., Massilon, Ohio.

Alderton, Asso. Prof. Nina M. Mills Coll., Oakland, Calif.

Alexander, Prof. J. W. Princeton Univ., Princeton, N. J. 29 Cleveland Lane.

ALGER, P. L. Electrical Engineer, General Electric Co., Schenectady, N. Y. 1758 Wendell Ave.

Allen, Dr. Bess Eversuld. (Mrs. C. E.). Detroit, Mich. 12073 Stoepel Ave. Allen, Asst. Prof. E. B. Rensselaer Polytechnic Inst., Troy, N. Y. 4 Sheldon Ave Allen, Asso. Prof. E. S. Iowa State Coll., Ames, Ia.

AlleN, Dr. Florence E. Instr., Univ. of Wisconsin, Madison, Wis. 219 Lathrop St.

Allen, Asso. Prof. Josepr. Coll. of the City of New York, New York, N. Y. ALLFN, Dr. MiLDRED. Research Inst., Oberlin Coll., Oberlin, Ohio.

Allen, Prof. R. B. Kenyon Coll., Gambier, Ohio.

Allison, Prof. Eli. Franklin School, New York, N. Y.

Alsberg, Dr. C. L. Director Food Research Inst., Stanford Univ., Stanford University, Calif. Box 1498 .

Alsberg, Julius. Consulting Engineer. 30 Church St., New York, N. Y.

\$AltmaN, F. Eastman Kodak Co., Rochester, N. Y. 1447 St. Paul St.

Ames, Prof. L. D. Univ. of Southern California, Los Angeles, Calif. $1328 \mathrm{~W} .37 \mathrm{th}$ Drve.

Amick, Prof. T. C. Elon Coll., Elon College, Alamance County, N. C.

Ananda-Rau, Prof. K. Presidency Coll., Madras, India.

Anderson, Dr. Nola L Instr, Univ of Missouri, Columbia, Mo. St. Catherine, Mo.

Anderson, Dr. Rose L. Instr., Hunter Coll., Park Ave. and 68th St., New York, N. Y. 1007 N. Main St., Jamestown, N. Y.

Anderson, Prof. W. E. Miami Univ., Oxford, Ohio.

Anderton, Asst. Prof. Ethel L. Mount Holyoke Coll., South Hadley, Mass.

ANDrews, Dr. ANNe D. B. Instr., Univ. of California, Berkeley, Calif. 65 Domıngo Ave.

\$ANDREws, Lucy. Missouri State Life Insurance Co., St. Louis, Mo.

Andrews, L. B. Harvard Univ., Cambridge, Mass. 54 Quint Ave., Boston, 34, Mass.

†Archibald, Prof. R. C. Brown Univ., Providence, R. I. 9 Charles Field St.

ArChibald, Asst. Prof. R. G. Columbia Univ., New York, N. Y. Hamilton Hall. Arms, Prof. R. A. Gettysburg Coll., Gettysburg, Pa. 143 Springs Ave

Armstrong, Dr. Beulah M. Instr, Univ. of Illinois, Urbana, Ill. 364 Math. Bldg.

Armstrong, Asst, Prof. L. E. Stevens Inst. of Technology, Hoboken, N. J. 
Arnetr, Ida M. Instr., Central Coll., McPherson, Kans. 922 S Maple St.

ArNold, Asst. Prof. H. E. Wesleyan Univ., Middletown, Conn. 264 Walliam St.

$\dagger$ ArNold, J. W. Electrical Engineer, Western Union Telegraph Co., 195 Broadway, New York, N. Y.

Artiaga, Santiago. City Engineer, Manila, P. I. City Hall.

Ashcraft, Prof. T. B. Colby Coll., Waterville, Me. 34 Pleasant St.

Astron, Prof. C. H. Univ. of Kansas, Lawrence, Kans. 1200 Ohio St.

\$ATANasofF, J. V. Instr., Univ. of Wisconsin, Madison, Wis.

†Atchison, Prof. C. S. Washington and Jefferson Coll., Washington, Pa. 442 E. Beau St.

Ater, Asst Prof. Muriel M. Univ. of Cincinnati, Cincinnati, Ohio.

AUde, Prof. H. T. R. Colgate Univ., Hamilton, N. Y.

\$Austin, Alice M. Instr., Univ. of Wisconsin, Madison, Wis.

AXEN, FLORENCE L. Instr., Univ. of Wisconsin at Milwaukee, Wis. 2320 Rowley Ave., Madison, Wis.

Ayers, FrANk. Box 53, Carlisle, Pa.

Ayers, Asso. Prof. W. L. Univ. of Michigan, Ann Arbor, Mich. 1225 White St.

Babb, Prof. M. J. Univ. of Pennsylvania, Philadelphia, Pa.

BABCOCK, Dean R. W. Kansas State Agricultural Coll., Manhattan, Kans.

BABCOCK, WeAlTHy. Instr., Univ. of Kansas, Lawrence, Kans.

†BACoN, Prof. Clara L. Goucher Coll., Baltimore, Md. $2316 \mathrm{~N}$ Calvert St.

BACON, H. M. Teaching Fellow, Stanford Univ., Los Angeles, Callf.

BAgBY, Prof. L. C. Linsly Inst. of Technology, Wheeling W. Va.

BaIDAFF, Dr. B. I. Univ. of Buenos Aires, Buenos Aires, Argentina. Belgrano 909.

Barley, Dean E. A. LaGrange Coll., LaGrange, Ga. 701 Vernon St.

BaILEY, Prof. F. H. Massachusetts Inst of Technology, Cambridge, Mass.

Balley, Dr. H. W. Associate, Univ. of Illinois, Urbana, Ill. 251 Math. Bldg.

BAKer, Asso. Prof. R. P. Univ. of Iowa, Iowa City, Ia. 929 Kurkwood Ave.

BALL, N. H. Instr., Massachusetts Inst. of Technology, Cambridge, Mass.

BalanTine, Dr. Constance R. (Mrs. J. P.). Associate, Univ. of Washington, Seattle, Wash. 4521 6th Ave., $N . E$.

Ballantine, Asso. Prof. J. P. Univ. of Washington, Seattle, Wash. 4521 th Ave., N. E.

BAmforth, Asst. Prof. F. R. Ohio State Univ., Columbus, Ohio.

BARDELL, R. H. Instr., Northwestern Univ., Evanston, Ill. 809 Simpson St.

Bareis, Asst. Prof. Grace M. Ohio State Univ., Columbus, Ohio. Canal Winchester, Ohro.

BARKER, J. E. Mabton, Wash.

Barnard, Asst. Prof. R. W. Univ, of Chicago, Chicago, Ill. 5426 Harper Ave.

\$BArNES, L. S. Instr., Lehigh Univ., Bethlehem, Pa.

Barnetr, Asso. Prof. I. A. Univ of Cincinnati, Cincinnati, Ohio.

Barninart, Prof C. A. Univ, of New Mexico, Albuquerque, N. Mex. $115 \mathrm{~S}$ Maple St., S. E.

Barone, H. G. Instr., Pennsylvania State Coll., State College, Pa.

§aArron, J. J. Univ. of Wisconsin, Madison, Wis. 405 North Hall.

Barrow, Prof. D. F. Univ. of Georgia, Athens, Ga. 436 Dearing St.

Barter, Dr. J. D. Box 66, Pacific Grove, Calif.

BartKy, Dr. Walter. Instr., Univ. of Chicago, Chicago, Ill.

Bartlett, Prof. D. P. Massachusetts Inst. of Technology, Cambridge, Mass. Basoco, Asst. Prof. M. A. Univ. of Nebraska, Lincoln, Neb.

§BAssford, Horace. Metropolitan Life Insurance Co., 1 Madison Ave., New York, N. Y.

Batchelder, Asso. Prof. P. M. Univ. of Texas, Austin, Tex. 808 W. $22 d$ St.

Bateman. Prof. Harry. California Inst. of Technology, Pasadena, Calif.

BAtEN, Dr. W. D. Instr., Univ. of Michigan, Ann Arbor, Mich.

BATTIG, LEON. Instr., Univ. of Wisconsin at Milwaukee, Milwaukee, Wis. 954 Buffum St

Bauer, P. S. Cruft Laboratory, Harvard Univ., Cambridge, Mass.

Beal, Asst. Prof. F. W. Univ. of Pennsylvania, Philadelphia, Pa. College Hall.

Beale, F. S. Instr., Univ. of Michigan, Ann Arbor, Mich.

BEAR, A. W. Univ. of Illinois, Urbana, Ill.

BEARD, Asst. Prof. H. L. Oregon State Coll., Corvallis, Ore. R. F. D. No. 2.

BeAtley, Asso. Prof. Ralph. Harvard Univ., Cambridge, 38, Mass. Harvard Graduate School of Education. 
Bearty, Asst. Prof. Samuel. Univ. of Toronto, Toronto, Canada

Beaty, Prof. E. B. 21 N. 27th St, Corvallis, Ore.

BECKETT, C. H. State Life Insurance Co., Indianapolis, Ind.

Beckwith, Prof. Ethelwynn R. (Mrs. W. E.). Milwaukee-Downer Coil., Milwaukee, Wis.

Beckwith, W. S. 731 Cobb St., Athens, Ga.

Beenken, Prof. May M. State Teachers Coll., Oshkosh, Wis.

BEETLE, Prof. R. D. Dartmouth Coll., Hanover, N. H.

BeIsel, Asst. Prof. B. R. Univ. of Pittsburgh, Pittsburgh, Pa. 72 Reppert Blud., Uniontown, $\mathrm{Pa}$.

Belcher, D. R. Asst. Chief Statistician, American Telephone and Telegraph Co., 195 Broadway, New York, N. Y.

Bell, Dr. Clifford. Instr., Univ. of California at Los Angeles, Los Angeles, Calif. 2414 Burnside Ave.

Bell, Prof. E. T. California Inst. of Technology, Pasadena, Calif.

Bell, Prof. Talmon. Sterling Coll., Sterling, Kans.

Bellamy, B. C. Civil Engineer. Box 438, Laramie, Wyo.

BENDER, Asst. Prof. H. A. Univ. of Akron, Akron, Ohio.

BENDer, Asst. Prof. J. R. Univ. of Idaho, Moscow, Id.

BENEDICT, Pres. H. Y. Univ. of Texas, Austin, Tex.

Benedict, Prof. Suzan R. Smith Coll., Northampton, Mass. 12 Barrett Place. †Bennetr, Prof. A. A. Brown Univ., Providence, R. I.

Bennetr, Asst. Prof. Theodore. Univ, of Wisconsin, Madison, Wis.

Benton, Dr. T. C. Instr., Yale Univ., New Haven, Conn. 17 Lake Place.

§BERG, Prof E. J. Union Coll., Schenectady, N. Y. Consulting Engineering Dept., General Electric Co.

Berkeley, L. M. Lawyer. 36 W. 91st St., New York, N. Y.

Berman, Benjamin. $253 \mathrm{~W}$. 73d St., New York, N. Y. Level Club

Bernstein, Prof. B. A. Univ. of California, Berkeley, Calif. 2785 Shasta Road.

Bernstorf, Prof. Ella E. Friends Univ., Wichita, Kans.

BerRy, Dr. A. C. National Research Fellow. Brown Univ., Providence, R. I. 550 Hope St.

Berry, Prof. E. M. Lynchburg Coll., Lynchburg, Va. 504 Westwood Ave.

Berry, William James. 18 Seaton Place, N. W., Washington, D. C.

Berry, Prof. Writiam Johnston. Brooklyn Polytechnic Inst., Brooklyn, N. Y. 224 St. John's Place.

Berwick, Prof. W. E. H. University Coll., Bangor, N. Wales, England. The Library.

Betz, Asst. Prof. Herman. Univ. of Missouri, Columbia, Mo.

Betz, William. 652 Melville St., Rochester, N. Y.

BeVERIDGE, Prof. H. R. Monmouth Coll., Monmouth, Ill. 316 Ninth St.

$\S$ BEWLEY, L. V. Engineering-General Dept., General Electric Co., Pittsfield, Mass. Bhattacharyya, Prof. D. P. Bareilly Coll., Bareilly, India.

BibB, Asst. Prof. S. F. Armour Inst. of Technology, Chicago, Ill.

Bieberbach, Prof. Ludwig. Univ. of Berlin, Berlin, Germany. Gelfertstrasse 16, Berlin-Dahlem.

BigbeE, J. A. 3110 Battery St., Little Rock, Ark.

BiggerstafF, John. Instr., State Coll. of Washington, Pullman, Wash.

BıkLÉ, C. E. Central High School, Syracuse, N. Y.

BIRCHBY, W. N. Instr., California Inst. of Technology, Pasadena, Calif.

Birchenougr, Prof. Harry. State Coll. for Teachers, Albany, N. Y.

Birkhoff, Prof. G. D. Harvard Univ., Cambridge, Mass. 984 Memoral Drive.

\$BISCAY, C. M., Sr. Western and Southern Life Insurance Co., 4th and Broadway, Cincinnati, Ohio.

§BISCAY, C. M., Jr. Western and Southern Life Insurance Co., Home Office Agency, 5711 Glengate Lane, Cincinnati, Ohio.

\$ishop, F. M. Eastman Kodak Co., Rochester, N. Y. 166 Nunda Blvd.

BLACK, A. H. Instr., Marietta Coll., Marietta, Ohio. 306 Warren St.

BlaCK, Dr. FloRenCE L. Instr., Univ. of Kansas, Lawrence, Kans.

Black, Prof. H. L. Westminster Coll., New Wilmington, Pa.

§LACK, J. E. Babcock and Wilcox Co., 85 Liberty St., New York, N. Y.

Blatr, Prof. HARold. Western State Normal Coll., Kalamazoo, Mich. 1220 Academy St.

BLAIR, VeVIA. Horace Mann School, New York, N. Y. 509 W. 121st St.

Blake, Dr. E. M. Drawer A, Pratt Station, Brooklyn, N. Y. 
Blichfeldt, Prof. H. F. Stanford Univ., Stanford Unıversity, Calif.

†Buiss, Prof. G. A. Univ. of Chicago, Chicago, Ill. 5625 Kenwood Ave.

Burss, W. L. Electrical Engineer, U. S. Light and Heat Corporation, Niagara Falls, N. Y. 142 Buffalo Ave.

Blue, Prof. A. H. Western Union Coll., Le Mars, Ia.

Buumberg, Prof. Henry. Ohio State Univ., Columbus, Ohio. $76 \mathrm{E}$. Blake Ave. Blumenthal, Dr. L. M. Instr., Rice Institute, Houston, Tex.

Bobertz, W. E. Research Engineer, Westinghouse Electric Co., Wilkinsburg, Pa. 556 Trenton Ave.

Bogard, Prof. Augustus. Coll. of St. Theresa, Winona, Minn 1018 W. 7 th St.

Bolza, Prof. Oskar. Univ. of Freiburg, Freiburg i. Br., Germany. Luisenstrasse 3.

Bond, Prof. J. D. Univ. of Tennessee, Knoxville, Tenn. Box 4217.

BoND, Prof. W. M. Waynesburg Coll., Waynesburg, Pa.

BoND, W. V. Univ. of Gettingen, Gcttingen, Germany. Weenderlandstr. 46.

Boothroyd, Prof. S. L. Cornell Univ., Ithaca, N. Y. Warley Place, R. R. No. 1. Borger, Prof. R. L. Ohio Univ., Athens, Ohio. P. O. Box 4217.

§BorofSky, SAmuel. Instr., Columbia Univ., New York, N. Y. 1483 E. $172 d$ St.

§oschwit, Bertea. Hunter Coll., New York, N. Y. 1049 Park Ave.

$\$$ Boss, W. D. Pacific Mutual Life Insurance Co., Los Angeles, Calif.

Boulad, FARID. Engineer, Bridges Dept., State Railways, Cairo, Egypt.

Bowden, Prof. JosePh. Adelphi Coll., Brooklyn, N. Y. 11 Brompton Road, Garden City, N. Y.

Bowen, Asst. Prof. L. H. Furman Univ., Greenville, S. C.

BowER, JuLIA W. Instr., Sweet Briar Coll., Sweet Briar, Va. Box 246.

\$BowER, Dr. O. K. Instr., Univ. of Illinois, Urbana, Ill.

Bowles, Asst. Prof. C. F. South Dakota State School of Mines, Rapid City, S. Dak.

§Boyajian, Aram. General Electric Co., Pittsfield, Mass.

Boyce, Dr. M. G. Instr., Adelbert Coll., Western Reserve Univ., Cleveland, Ohio.

BOYD, Prof. P. P. Univ. of Kentucky, Lexington, Ky.

BoydsDon, Asst. Prof. R. W. Miami Univ., Oxford, Ohio. Physics Dept.

Bradshaw, Prof. J. W. Univ. of Michigan, Ann Arbor, Mich. 1304 Cambradge Road.

§BRadT, PaUL. Junior Examiner, Patent Office, Washington, D. C.

§BRADY, G. V. Metropolitan Life Insurance Co., 1 Madison Ave., New York, N. Y.

Brahana, Asst. Prof. H. R. Univ. of Illmois, Úrbana, Ill.

BRAMbLe, Prof. C. C. U. S. Naval Academy, Annapolis, Md. 85 5th St.

BRAND, F. J. Instr., Univ. of British Columbia, Vancouver, Canada.

BRAND, Prof. LouIs. Univ. of Cincinnati, Cincinnati, Ohio.

BRANDEBerRY, Prof. J. B. Univ. of Toledo, Toledo, Ohio.

Branovan, LEo. Instr., Univ. of Minnesota, Minneapolis, Minn.

Brant, Prof. Laura. Ouachita Coll., Arkadelphia, Ark.

BRASEFIELD, Prof. S. E. Rutgers Univ., New Brunswick, N. J.

Bratton, Prof. W. A. Whitman Coll., Walla Walla, Wash. 570 Boyer St.

BRAY, Asst. Prof. H. E. Rice Inst., Houston, Tex.

Breckenridge, W. E. Associate, Columbia Univ., New York, N. Y. 21 Sycamore Ave., Mount Vernon, N. Y.

BreIt, Dr. Gregory. Dept. of Terrestrial Magnetism, Carnegie Institution, Washington, D. C. 36th St. and Broad Branch Road.

BRENKE, Prof. W. C. Univ. of Nebraska, Lincoln, Neb.

Briggs, Dr. G. B. Hun School, Princeton, N. J.

BRIGHT, EDward. Sandwich, Cape Cod, Mass.

BRILL, John. 75 Seymour St., Marble Arch, London, W. 2, England.

BrinK, Prof. R. W. Univ. of Minnesota, Minneapolis, Minn. 55 Williams Ave, $S . E$.

Brinkmann, Asst. Prof. H. W. Harvard Univ., Cambridge, Mass. 44 Concord Ave.

§BrIston, W. A. Instr., Univ. of Pennsylvania, Philadelphia, Pa. 515 Runnymede Ave., Jenkintown, Pa.

Brixey, J. C. Instr., Univ. of Oklahoma, Norman, Okla. 303 University Blvd.

BRodIE, Prof. W. M. Virginia Polytechnic Inst., Blacksburg, Va.

Brooke, Prof. W. E. Univ. of Minnesota, Minneapolis, Minn.

Brown, Dr. A. B. Instr., Columbia Univ., New York, N. Y. 605 Furnald Hall.

BRown, Asst. Prof. B. H.' Dartmouth Coll., Hanover, N. H.

Brown, B. L. Instr., Amherst Coll., Amherst, Mass. 34 Amity St.

Brown, Dr. Eleanor P. (Mrs. B. H.). Hanover, N. H. 
†Brown, Prof. E. W. Yale Univ., New Haven, Conn. 116 Everit St.

Brown, Prof. H. S. Hamilton Coll, Clinton, N. Y. College Campus.

Brown, Dr. O. E. Instr., Case School of Applied Science, Cleveland, Ohio. 1754 Noble Road.

Browne, Asso. Prof E. T. Univ. of North Carolina, Chapel Hill, N. C. $615 F$ Franklin St.

Brownstein, Benjamin. 5174 th St, Ellwood City, Pa.

Bruce, Prof. R. E. Boston Univ., Boston, Mass. 688 Boylston St.

Brumbaugh, Prof M. A. Bureau of Business and Social Research, Univ. of Buffalo. Buffalo, $N$ Y.

$\S B$ Rush, R. G. Prudential Insurance Co , Newark, N. J.

Bryan, Asso Prof. N. R. Univ. of Maine, Orono, Me. 32 Myrtle St.

Buchanan, Dean Daniel. Univ, of British Columbia, Vancouver, Canada.

Buchanan, Prof H. E. Tulane Univ, New Orleans, La.

Buck, Asso. Prof. Thomas. Univ. of California, Berkeley, Calif. Faculty Clu'q

Budd, B. I. Chicago, North Shore and Milwaukee Railroad Co., 72 W. Adams St., Chicago, Ill.

Bullard, Prof. J. A. Univ. of Vermont, Burlington, Vt. 110 Summit St.

\$BulleR, F. H. General Electric Co., Schenectady, N. Y. 1095 Brerwood Blvd. Bullite, W. M. Lawyer. 1711 Inter Southern Bldg., Louisville, Ky.

Bullock, R. C. Instr., Univ, of North Carolina, Chapel Hill, N. C.

Bumer, Asst. Prof. C. T. Ohio State Univ., Columbus, Ohio.

Bunnel,, C. T. Instr., Univ. of Rochester, Rochester, N. Y. 88 Richmond St.

Bunyan, L. H. Instr., Univ. of Wisconsin at Milwaukee, Milwaukee, Wis. 619 State St.

†Burgess, Dr. H. T. Morningside, Milford, Conn.

Burgess, Dr. R. W. Chief Statistician, Western Electric Co., 195 Broadway, New York, N. Y.

\$Burian, O. J. Missouri State Life Insurance Co., St. Louis, Mo.

Burington, R. S. Instr., Case School of Applied Science, Cleveland, Ohio.

BurketT, Asst. Prof. F. J. H. Union Coll., Schenectady, N. Y.

Burnam, Prof. J. E. Simmons Univ., Abilene, Tex. 1846 Orange St.

Burns, Dr. KeIvis. Allegheny Observatory, Pittsburgh, Pa.

Burton, H. E. Principal Astronomer, U S Naval Observatory, Washington, D. C. Burwell, Dr. W. R. Continental Share Inc., 520 Cuyahoga Bldg., Cleveland, Ohio.

BusH, L. E. 19 Berkeley Place, 2112 N. High St., Columbus, Ohio.

Bushey, J. H. Instr., Univ. of Michigan, Ann Arbor, Mich. 1015 Church St.

Bussey, Prof. W. H. Univ. of Minnesota, Minneapolis, Minn.

Butler, L. G. Instr., State Coll. of Washington, Pullman, Wash.

Byerly, Prof. W. E. Harvard Univ., Cambridge, Mass. 39 Hammond St.

ByrNE, Dr. W. E. Box 104, Station B, Hattiesburg, Miss.

Cain, Prof. Wrlliam. Univ. of North Carolina, Chapel Hill, N. C.

CaIrns, S. S. Instr., Yale Univ., New Haven, Conn. 123 Pendleton St.

Carrns, Prof. W. D. Oberlin Coll., Oberlin, Ohio. Peters Hall.

Calmoun, Prof. J. W. Univ. of Texas, Austin, Tex. 2805 Rro Grande St.

Calkins, Prof. Helen. Pennsylvania Coll. for Women, Pittsburgh, Pa.

Callahan, Prof. Ethel, B. Cedar Crest Coll., Allentown, Pa.

Calloway, Prof. Theodosia T. (Mrs.). Stephens Coll., Columbia, Mo. Dumas Apts.

Cameron, E. A. Instr., Univ. of North Carolina, Chapel Hill, N. C.

Cameron, R. H. Instr., Cornell Univ., Ith ca, N. Y. Whate Hall.

†Camp, Prof. B. H. Wesleyan Univ., Middletown, Conn. $110 \mathrm{Mt}$ Vernon St.

Camp, Prof. C. C. Univ. of Nebraska, Lincoln, Neb.

Campbell, Prof. A. D. Syracuse Univ., Syracuse, N. Y. 310 Kensington Road.

Campbeld, Prof. D. F. Armour Inst. of Technology, Chicago, Ill.

Campbell, Dr. G. A. American Telephone and Telegraph Co., 195 Broadway, New York, N. Y.

CAMpbell, Prof. J. W. Univ, of Alberta, Edmonton, Canada.

Campbell, W. B. Lecturer, Judson Coll., Rangoon, Burma.

Candy, Prof. A. L. Univ. of Nebraska, Lincoln, Neb. Station $A$.

Capron, Prof. Paul. U. S. Naval Academy, Annapolis, Md.

Carey, Asso. Prof. E. F. A. Univ. of Montana, Missoula, Mont.

CARIs, Asst. Prof. P. A. Univ. of Pennsylvania, Philadelphia, Pa. 717 Runnymede Ave., Drexel Hill, Pa. 
SCarlen, Mildred E. Brown Univ., Providence, R. I.

\$CARLITZ, Leonard. National Research Fellow. California Inst. of Technology, Pasadena, Calif.

Carlson, Asst. Prof. Elizabeth. Univ. of Minnesota, Minneapolis, Minn. 3020 14th Ave., S.

Carman, Prof. M. G. Murray State Teachers Coll., Murray, Ky. College Station, Box 535.

Carmichael, G. N. Instr., Brown Univ., Providence, R. I.

CARMiChael, Prof. R. D. Univ. of Illinois, Urbana, Ill. 207 W. Washington Blvd.

CARPENTER, Prof. A. F. Univ. of Washington, Seattle, Wash.

CARPENTER, R. V. Metropolitan Life Insurance Co., 1 Madison Ave., New York, N. Y.

CARr, Prof. E. L. Union Univ., Jackson, Tenn. 109 Camden St.

CARR, Prof. F. E. Oberlin Coll., Oberlin, Ohio. 284 Forest St.

CARrie, R. A. Tutor, Coll. of the City of New York, New York, N. Y. Furnald Hall, Columbia Unv.

\$Carrington, J. R. L. Union Central Life Insurance Co., Cincinnati, Ohio. Room 1120, Union Central Life Bldg.

Carroll, Asst. Prof. Evelyn T. Wells Coll., Aurora, N. Y.

Carroll, Asst Prof. I. S. Syracuse Univ., Syracuse, N. Y. 511 Comstock Ave.

†Carruth, Prof. W. M. Hamilton Coll., Clinton, N. Y. College Hall.

CARSLAw, Prof. H. S. Univ. of Sydney, Sydney, Australia.

CARson, J.R. American Telephone and Telegraph Co., 195 Broadway, New York, N. Y.

Carter, C. C. Lawyer. Bluffs, Scott Co., Ill.

CARTER, E. R. National Life Insurance Co. of the U. S. A., Chicago, 111. 29 S. La Salle St.

Carter, H.C. Instr., Univ. of Missouri, Columbia, Mo. 213 Engineernng Bldg.

Carus, Dr. E. H. La Salle, ill.

§Carus, Mrs. MARY H. La Salle, Ill.

Carver, Prof. W. B. Cornell Univ., Ith-ca, N. Y. White Hall.

CARY, R. L. Writer. Prinz-Louis-Ferdınand-Str. 5, Berlin N. W. 7, Germany.

\$CAssidy, P. R. Fuller Lehigh Co., Fullerton, Pa.

Castellant, Dr. Maria. Via Capo Peloro 3, Monte Sacro, Rome, Italy.

CAstle, S. N. Engineer. 655 Park Ave., New York, N. Y.

Cenerberg, Prof. W. E. Augustana Coll., Rock Island, Ill. $2542221 / 2$ Ave.

Chase, Dr. A. B. Chancellor, Brown Univ., Providence, R. I. $9 g$ Power St.

Chafee, Prof. Zechariah. Harvard Univ., Cambridge, Mass. Harvard Larw School.

Crambers, Prof. G. G. Univ. of Pennsylvania, Philadelphia, Pa. 251 S. 38th St.

Chang, F. H. L. Instr., Nankai Univ., Tientsin, China.

Chapelon, Prof. JACQues. Univ. of Lille, Lille, France. 2 Boulevard Morland, Parzs.

Cheney, Prof. W. F. Connecticut Agric. Coll., Storrs, Conn.

\$Chenowetr, D. K. Western and Southern Life Insurance Co., 4th and Broadway, Cincinnati, Ohio.

Chiang, Prof, Tso. Nankai Univ., Tientsin, China.

Chittenden, Prof. E. W. Univ. of Iowa, Iowa City, Ia. 221 Physics Bldg.

Christianson, L. C. Instr., Texas Technological Coll., Lubbock, Texas.

\$Christman, J. A. Actuary, Metropolitan Life Insurance Co,, 1 Madison Ave., New York, N. Y.

Church, Asst. Prof. Alonzo. Princeton Univ., Princeton, N. J. 25 Murray Place. Church, EarL. Parish, N. Y.

Church, W. R. The Peddie School, Hightstown, N. J. 208 Mattison Ave., Ambler, $P a$.

Churchrli, Asst Prof. R. V. Univ. of Michigan, Ann Arbor, Mich. 28 Rosewood St.

Clark, Asst. Prof. A. G. Colorado Agricultural Coll., Fort Collins, Colo.

ClARK, J. A. 114 Sage Place, Ithaca, N. Y.

Clarke, Prof. E. H. Hiram Coll, Hiram, Ohio.

CLARKSON, J. M. Instr, Cornell Univ, Ithaca, N. Y.

Clawson, H'rof $\mathrm{I}$ W. Úrsinus Coll., Collegeville, Pa. 6 Glenwood Ave.

Clements, Prof. G. R. U. S. Naval Academy, Annapolis, Md. 7 Thompson St.

\$Clifford, Prof. H. E. Harvard Univ., Cambridge, Mass. Pierce Hall.

ClUTz, Prof. F. H. Gettysburg Coll., Gettysburg, Pa. 159 Broadway.

Coвb, Prof, C. W. Amherst Coll., Amherst, Mass. 75 S. Pleasant St. 
Coвb, Prof. H. E. Lewis Inst., Chicago, Ill.

CoBle, Prof. A. B. Univ. of llinois, Urbana, Ill. $702 \mathrm{~W}$. Washington Blvd.

CoE, Asst. Prof. C. J. Univ, of Michigan, Ann Arbor, Mich. 524 Linden Ave. Coffin, Prof. L. M. Coe Coli., Cedar Rapids, Ia. 1027 2d Ave.

Cohen, Prof. Abraham. Johns Hopkins Univ., Baltimore, Md.

Conen, L. W. Instr., Univ. of Michigan, Ann Arbor, Mich. 3018 Angell Hall.

Cohen, Asst. Prof. Treresa. Pennsylvania State Coll., State College, Pa. 1709 Linden Ave., Baltimore, Md.

Cole, Dr. Margaret B. (Mrs H. O.). 72 Wilson Ave., Morgantown, W. Va.

Cole, Nancy. 263 Sandwich St., Plymouth, Mass.

Colmman, Prof. J. B. Univ. of South Carolina, Columbia, S. C.

Colliter, Dr. MYrTiE. 3201/2 N. Alexander Ave., Los Angeles, Calif.

Collignon, Dr. G. H. Box 57, Durham, N. H.

Collingwoon, Dr. E. F. Trinity Coll., Cambridge, England.

†Collıns, O. C. Instr., Univ. of Nebraska, Lincoln, Neb. $1920 \mathrm{~S} .26 \mathrm{th} \mathrm{St}$.

ColpITrs, Asso. Prof. E. C. State Coll. of Washington, Pullman, Wash. 510 Maiden Lane.

Colpitrs, Asso. Prof. Julia T. Iowa State Coll., Ames, Ia. 29 Cranford Apts.

Colwell, Prof. R. C. West Virginia Univ., Morgantown, W. Va.

Col Yer, Prof. E. E Kansas State Teachers Coll., Hays, Kans.

Comegys, Esther. Harvard Univ., Cambridge, Mass. 21 Channcey St.

Comstock, Prof. C. E. Bradley Polytechnic Inst., Peoria, Ill.

Congdon, Asso. Prof. A. R. Univ. of Nebraska, Lincoln, Neb. Teachers College, Columbia Univ, Nerw York, N. Y.

Conkling, Prof. R. P. Central High School, Newark, N. J. 31 N. 10th St.

Conkwright, Asst. Prof. N. B. Univ. of Iowa, Iowa City, Ia. 127 Physics Bldg.

Conran, Prof. M. J. University Coll., Cork, Ireland.

Conwell, Dr. G. N. St. Paul's School, Concord, N. H.

Conwelt, Prof. H. H. Beloit Coll., Beloit, Wis. 1621 Emerson St.

Cook, Asso. Prof. A. J. Univ. of Alberta, Edmonton, Canada.

Cook, Asst. Prof. L. T. St. Lawrence Univ., Canton, N. Y. 15 Harrison St.

Cooley, H. R. Instr., New York Univ., New York, N. Y. 100 Washington Square East.

†Coolidge, Prof. J. L. Harvard Univ., Cambridge, Mass. 50 Holyoke St.

Cooper, Dr. Elizabeth M. Principal, Buckingham School, Cambridge, Mass. 2 Buckingham St.

\$COoper, L. J. Asst. Actuary, Pacific Mutual Life Insurance Co., Los Angeles, Calif.

Cope, Asst. Prof. T. F. Marietta Coll., Marietta, Ohio.

Copeland, Asst. Prof. A. H. Univ. of Michigan, Ann Arbor, Mich.

†Copezand, Asso. Prof. Lennie P. Wellesley Coll., Wellesley, Mass. 14 Waban St.

Copp, P. T. Valparaiso Univ., Valparaiso, Ind.

Coral, Max. Univ of Chicago, Chicago, Ill.

Corbin, Prof. C. E. Coll. of the Pacific, Stockton, Calif. 117 W. Euclid.

Corliss, J. J. Instr., Univ. of Michigan, Ann Arbor, Mich. 1014 Cornvell Place.

Cosby, Prof. Byron. State Teachers Coll., Kirksville, Mo.

Court, Asso Prof. N. A. Univ. of Oklahoma, Norman, Okla. 425 W. Eufaula St.

Cowlex, Prof. Elizabeth B. Vassar Coll., Poughkeepsie, N. Y. 913 Arch St., North Side, Pittsburgh, Pa.

Cox, Asso. Prof. E. F. Howard Univ., Washington, D. C.

Cox, Dr. L. C. 2153 N. Capitol Ave., Indianapolis, Ind.

CraIG, Dr. C. C. Instr., Univ. of Michigan, Ann Arbor, Mich. 1509 Brooklyn Ave.

CRAIG, Asst. Prof. C. F.' Cornell Univ., Ithaca, N. Y. 311 Elmwood Ave.

Craig, H. V. Adjunct Prof., Univ, of Texas, Austin, Tex.

Craig, J. D. Metropolitan Life Insurance Co., 1 Madison Ave., New York, N. Y.

Cramblet, Prof. W. H. Bethany Coll., Bethany, W. Va.

CRAMER, PAul. Instr., Univ. of Arkansas, Fayetteville, Ark.

Cramler, Asst. Prof. C. M. Univ. of Washington, Seattle, Wash. Philosophy Hall.

Crane, Asso. Prof. Rufus. Ohio Wesleyan Univ., Delaware, Ohio. 39 Montrose Ave.

Crathorne, Asso. Prof. A. R. Univ. of Illinois, Urbana, I1l. 802 Pennsyluania Ave.

CRAwlex, Prof. E. S. Univ. of Pennsylvania, Philadelphia, Pa. College Hall.

CRESSE, Prof. G. H. Univ. of Arizona, Tucson, Ariz.

Crockett, Prof. C. W. Rensselaer Polytechnic Inst., Troy, N. Y. 
Cromweld, J. W. 1815 13th St., Washington, D. C.

Crout, P. D. 219 23d St., Milwaukee, Wis.

Crum, Prof. W. L. Harvard Univ., Cambridge, Mass. Economics Dept. R. F. D. Sunderland, Arlington, Vt.

Cummings, H.K. Bureau of Standards, Washington, D. C. 4213 Ellicott St., N.W., Friendship Station.

Cummings, Prof. Louise D Vassar Coll, Poughkeepsie, N Y

CuRIEL, H. W. Illinois Life Insurance Co., Chicago, Ill. 1212 Lake Shore Drive.

Currier, A. E. Instr., Harvard Univ., Cambrige, Mass. 36 Conant Hall.

Currier, Asso. Prof. C. H. Brown Univ., Providence, R. I.

Curry, Asst. Prof. H. B. Pennsylvania State Coll., State College, Pa. 805 W. Beaver Ave.

CurTis, A. M. State Normal School, Oneonta, N. Y.

CurTis, Prof. H. B. Lake Forest Coll., Lake Forest, Ill. 11 College Campus.

CurTIs, Dr. H. D. Director, Allegheny Observatory, Pittsburgh, Pa.

CurTiss, Prof. D R. Northwestern Univ., Evanston, '11.

CuTler, Dr. E. H. Instr., Lehigh Univ., Bethlehem, Pa.

Dadourian, Prof. H. M. Trinity Coll., Hartford, Conn. 125 Vernon St.

DALAKER, Prof. H. H. Univ. of Minnesota, Minneapolis, Minn. 523 Walnut St., $S . E$.

Dancer, Asst. Prof. Wayne. Toledo Univ., Toledo, Ohio. 1929 Otiarva Drive.

Daniell, Prof. P. J. Sheffield Univ., Sheffield, England.

Daniells, Asst. Prof. Marian E. Iowa State Coll., Ames Ia. Box 332, Station A.

Dantzig, Asst. Prof. Tobias. University of Maryland, College Park, Md. 1662 Park Road, N. W., Washington, D. C.

Darkow, Asst. Prof. Marguerite D. Hunter Coll., Park Ave. and 68th St., New York, N. Y.

DARLING, F. W. U. S. Coast and Geodetic Survey, Washington, D. C.

Daus, Asso. Prof. P. H. Univ. of California at Los Angeles, Los Angeles, Calıf.

Davis, C. H. 130 Retreat Ave., Hartford, Conn.

Davis, Asst. Prof. D. R. Univ. of Oregon, Eugene, Ore. 1263 Oak St.

Davis, Asst. Prof. H. A. Univ. of West Virginia, Morgantown, W. Va. 461 Virginia Ave.

†DAvis, Pres. H. N. Stevens Inst. of Technology, Hoboken, N. J.

Davis, Asso. Prof. H. T. Indiana Univ., Bloomington, Ind.

Davrs, Asso. Prof. J. E. Drexel Inst., Philadelphia, Pa. $32 d$ and Chestnut Sts.

Davis, Prof. J. M. Univ. of Kentucky, Lexington, Ky. 340 Madison Place. Dean, Alice C. Rice Inst., Houston, Tex.

Decherd, Adj. Prof. Mary E. Univ. of Texas, Austin, Tex. 2313 Nueces St. DEck, Prof. L. J. Muhlenberg Coll., Allentown, Pa. 232 N. 15th St.

DECKER, Prof. F. F. Syracuse Univ., Syracuse, N. Y. 312 Marshall St.

De Cleene, Prof. L. A. V. St. Norbert Coll., West De Pere, Wis.

DECou, Prof. E. E. Univ. of Oregon, Eugene, Ore. 929 Hilyard St.

$\nmid$ DEDERICK, Lr. L. S. Aberdeen Proving Ground, Aberdeen, Md.

Defoe, Prof. L. M. Univ. of Missouri, Columbia, Mo. 810 Virginia Ave.

De Foe, Prof. O. K. Coll. of the Ozarks, Clarksville, Ark.

DenN, Dr. Edgar. Instr., Columbia Univ., New York, N. Y. 304 Hamilton Hall. DEIMEL, Asso. Prof. R. F. Stevens Inst. of Technology, Hoboken, N. J.

†DELong, Prof. I. M. Univ, of Colorado, Boulder, Colo. 517 Pine St.

DeLury, Prof. A. T. Univ. of Toronto, Toronto, Canada.

Deming, Prof. R. M. Upper Iowa Univ., Fayette, Ia.

Dkmos, Dr. M. S. Instr., Columbia Univ., New York, N. Y. 823 Furnald Hall.

\$DenHartog, J. Westinghouse Electric Co., E. Pittsburgh, Pa.

Denton, Asst. Prof. W. W. Univ. of Michigan, Ann Arbor, Mich. 1014 Cornwell Place.

Derby, Lieut. G. T. Corps of Engineers, U. S Army. Fort DuPont, Del.

Dickrnson, Prof. C. N. Hollins Coll., Hollins, Va.

Dickson, Prof. L. E. Univ. of Chicago, Chicago, Ill. 5535 Universaty Ave.

DicksteIn, Prof. S. Univ. of Warsaw, Warsaw, Poland. Marzalkowska 117.

Dillingham, Prof. Alexander. U. S. Naval Academy, Annapolis, Md.

Dimick, Prof. C. E. U. S. Coast Guard Academy, New London, Conn.

Dines, Prof. L. L. Univ, of Saskatchewan, Saskatoon, Canada.

Dixon, Prof. A. L. Magdalen Coll., Oxford, England.

Drxov, E. T. Billy Dun, Jamaica, British West Indies. 
Doak, Prof. Eleanor C. Mount Holyoke Coll., South Hadley, Mass. Faculty House.

DoBert, Asst. Prof. H. A. New York State Coll. for Teachers, Albany, N. Y. Dodn, Prof. E. L. Univ. of Texas, Austin, Tex. 3012 West Ave.

DoERMaNN, Dr. F. W. Instr., New York Univ., University Heights, New York, N. Y.

Donahue, Asso. Prof. J. E. Univ. of Vermont, Burlington, Vt. Essex Junction.

Doner, Asso. Prof. R. D. Alabama Polytechnic Inst., Auburn, Ala. Box 2235.

Doole, Dr. H. P. Instr., Univ. of Nebraska, Lincoln, Neb.

DORROH, J. L. Instr., Univ. of Texas, Austin, Tex. Box 1667, University Station.

DoRWarT, H. L. Instr., Williams Coll., Williamstown, Mass. Faculty Club. \$DorweIler, Patr. Actuary, Aetna Life Insurance Co., Hartford, Conn.

Dostal, Asst. Prof. B. F. Univ. of Florida, Gainesville, Fla.

Douglas, Asst. Prof. JEsse. Massachusetts Inst of Technology, Cambridge, Mass.

DownIng, Asso. Prof. H. H. Univ. of Kentucky, Lexington, Ky. 138 State St.

\$DoxSEE, M. I. Aetna Life Insurance Co., Hartford, Conn.

Dresden, Prof. ARnold. Swarthmore Coll., Swarthmore, Pa. 606 Elm Ave.

\$DUERKSEN, J. A. Coast and Geodetic Survey, Washington, D. C.

Duncan, Dr. D. C. Instr., Univ. of California, Berkeley, Calif. 420 Wheeler Hall.

D'Unger, V. C. Lincoln National Life Insurance Co., Ft. Wayne, Ind. $910 \mathrm{~W}$. Berry St.

Dunkel, Asso. Prof. Otro. Washington Univ., St. Louis, Mo.

DunlaAp, L. T. Instr., Pennsylvania State Coll,, State College, Pa.

†Durand, Prof. W. F. Stanford Univ., Stanford University, Calif.

Durell, Dr. Fletcher. Belleplain, N. J.

DureN, Dr. W. L., JR. Instr., Coll. of the City of Detroit, Detroit, Mich.

DurfeE, Prof. W. H. Hobart Coll., Geneva, N. Y. 403 Pulteney St.

DURFEe, Prof. W. P. Hobart Coll., Geneva, N. Y.

\$Dushman, Dr. Saul. Research Laboratory, General Electric Co., Schenectady, N. Y.

Dushnik, Bes. Instr., Univ. of Michigan, Ann Arbor, Mich. 1930 Cambridge Road.

Dwyer, Asst. Prof. P.S Antioch Coll., Yellow Springs, Ohio.

Dye, Dr. L. A. Instr., Cornell Univ., Ithaca, N. Y. White Hall.

Dysart, RoBert. Certified Public Accountant. 40 State St., Boston, Mass.

EAGLes, Prof. T. R. Howard Coll., Birmingham, Ala. 8016 2d Ave. S.

EARL, Dr. J. M. Instr., Univ. of Iowa, Iowa City, Ia.

EARLE, Prof. M. D. Furman Univ., Greenville, S. C.

Echols, Prof. C. P. U.S. Military Academy, West Point, N. Y.

Echols, Prof. W. H. Univ. of Virginia, University, Va.

ECkERSLEY, J. O. Consulting Engineer. 4269 White Plains Ave., New York, N. Y. ECKLes, AlLegra. State Farm, Niantic, Conn.

EDIngton, Prof. W. E. De Pauw Univ., Greencastle, Ind.

EDison, T. M. Research Engineer 106 N. Walnut St., East Orange, N. J.

EDMondson, Prof. T. W. New York Univ., University Heights, New York, N. Y. Edmonson, Asst. Prof. Nat. Texas Technological Coll., Lubbock, Tex.

EDWards, Prof. G. C. Univ. of California, Berkeley, Calif. 2546 Dana St.

EDWARDs, Asso. Prof. P. D. Ball State Teachers Coll, Muncie, Ind. $713 \mathrm{~W}$. North St.

Eide, Margaret C. (Mrs. R. B.). 308 S. Second St., River Falls, Wis.

EIESLANd, Prof. John. Univ. of West Virginia, Morgantown, W. Va. 361 Demain Ave.

†EIsenhart, Prof. L. P. Princeton Univ., Princeton, N. J.

EkMaN, W. E. Instr., Univ. of South Dakota, Vermilion, S. Dak.

\$ELDEN, L. L. Edison Electric Illuminating Co., 39 Boylston St., Boston, Mass.

EldER, Dr. J. D. Teaching Fellow. California Inst. of Technology, Pasadena, Calif.

Ellingson, H. E. Luther Coll., Decorah, Ia.

†Ellrotr, Prof. E. B. Oxford Ưniv., Oxford, England. 4 Bardwell Road.

ElliotT, Prof. W. W. Duke Univ., Durham, N. C. Box 533.

Ellis, G. A. 920 5th Ave., New York, N. Y.

§ELston, J. S. Travelers Insurance Co., Hartford, Conn.

EMCH, Prof. Arnold. Univ. of Illinois, Urbana, Ill. $1002 \mathrm{~S}$. Orchard St.

Emmons, Prof. C. W, Simpson Coll., Indianola, Ia, 
Emmons, Prof. L. C. Michigan State Coll., East Lansing, Mich.

ENGLISH, HarRY. Franklin School, 13th and K Sts., N. W., Washington, D. C. Engstrom, Dr. H. T. National Research Fellow. Göttingen Univ., Gottingen, Germany. 8 Washington St., Plymouth, Mass

ENTz, G. G. 1352 Poinsettia Place, Hollywood, Calif.

Epsteen, Dr. Saul. Tremont Hotel, Denver, Colo.

ERIKson, Asst. Prof. C. M. Michigan State Normal Coll., Ypsilanti, Mich. $311 \mathrm{~W}$. Cross St.

ERIKson, Robert. Univ. of Wisconsin, Madison, Wis. 609 Sheldon St.

ERwIN, Prof. J. T. George Washington Univ., Washington, D. C. 2023 G St., N. $W$.

†Escotт, E. B. Actuary. 1019 S. East Ave., Oak Park, Ill.

Eshleman, Prof. J. D. Heidelberg Coll., Tiffin, Ohio.

EsTy, Dean T. C. Amherst Coll., Amherst, Mass. 40 Dana St.

Etruinger, Prof. H. J. Univ, of Texas, Austin, Tex. 3110 Harris Park Ave.

Evans, A. A. Instr., Columbia Univ., New York, N. Y. 201 Hamilton Hall. †Evans, Prof. G. C. Rice Inst., Houston, Tex.

Evans, G. W. 107 Ocean St., Lynn, Mass.

Evans, Prof. H. B. Univ. of Pennsylvania, Philadelphia, Pa. College Hall.

Evans, Asst. Prof. H. P. Univ. of Wisconsin, Madison, Wis. North Hall.

Evans, P. H. Vice-Pres., Northwestern Mutual Life Insurance Co., Milwaukee, Wis. 210 E. Wisconsin St.

§Evans, R. D. Westinghouse Electric Co., E. Pittsburgh, Pa.

EveretT, Prof. H.S. Univ, of Chicago, Chicago, Ill.

Falconer, B. L. 315 Ward St., Marlin, Tex.

Fales, Asso. Prof. H. A. Columbia Univ., New York, N. Y. Havemeyer Hall.

Farnau, Prof. E. F. Univ. of Cincinnati, Cincinnati, Ohio Chemistry Dept.

FARNUM, Dr. FAY. Instr., Washington Square Coll., New York Univ., New York, N. Y.

FARRELL, Dr. O. J. Rockefeller International Research Fellow at Univ. of Munich, Germany. 109 Windsor St., Reading, $\mathrm{Pa}$.

Faught, Prof. J. B. Yankton Coll., Yankton, S. Dak.

FEInLER, Rev. F. J. 1414 East Ave., Akron, Ohio.

FELD, J. M. Instr., Columbia Univ., New York, N. Y. Furnald Hall.

FeltGeS, EdNa M. Instr., Crane Junior Coll., Chicago, Ill. 6437 Kimbark Ave.

FenN, Asso. Prof. I. H. Brooklyn Polytechnic Inst., Brooklyn, N. Y. 99 Livingston St.

Fenner, Beatrice A. South Barbara High School, Palo Alto, Calif. 1010 Forest Court.

†FErRy, Pres. F. C. Hamilton Coll., Clinton, N. Y.

FIELD, Prof. FloYD. Georgia School of Technology, Atlanta, Ga. R. F. D. 1, Box 38, Decatur, Ga.

FIeld, Prof. Peter. Univ. of Michigan, Ann Arbor, Mich. 904 Olivia Ave.

FIELD, S. E. Univ. of Michigan, Ann Arbor, Mich.

fFields, Prof. J. C. Univ. of Toronto, Toronto, Canada.

FINAN, Dr. E. J. Instr., Catholic Univ. of America, Washington, D. C.

FIndlay, Prof. Willtam. McMaster Univ., Hamilton, Ont., Canada.

FinkeI, Prof. B. F. Drury Coll., Springfield, Mo.

FISHER, ARne. Actuary, Western Union Telephone and Telegraph Co., 195 Broadway, New York, N. Y.

Fisher, Clatre B. Instr., New York Univ., New York, N. Y. 100 Washington Square E.

Fisher, Prof. Irving. Yale Univ., New Haven, Conn. 460 Prospect St.

\$Fisk, N. C. Instr., Univ. of Michigan, Ann Arbor, Mich. 1505 Morton Ave.

†Fiske, Prof. T. S. Columbia Univ., New York, N. Y.

FiTCH, Dr. ANNIE M. (Mrs. EDWARD). Clinton, $N$. $\dot{Y}$.

†Frre, Prof. W. B. Columbia Univ., New York. N. Y.

Fithian, Asst. Prof. J. H. Newark Coll. of Engineering, Newark, N. J. 147 Halsted St., E. Orange, $N$. J.

Fitterer, Prof. J. C. Colorado School of Mines, Golden, Colo. 1620 Maple St. \$FitzGerald, A. H. Asst. Actuary, Prudential Insurance Co., Newark, N. J. Flanders, Dr. D. A. Instr., New York Univ., University Heights, New York, N. Y. FLANDERS, Asst. Prof. R. L. Oklahoma Agric. and Mech. Coll., Stillwater, Okla. $212 \mathrm{Elm}$ St. 
Fleisher, Asst. Prof. Edward. Coll. of the City of New York, New York, N. Y. 1068 Park Place, Brooklyn, N. Y.

Fleisig, A. J. Instr., St. Procopius Coll., Lisle, Ill.

FleXNeR, W. W. Instr., Princeton Univ., Princeton, N J. Graduate Coll.

Furns, A. D. Director, Engineering Foundation, 29 W. 39th St., New York, N. Y. FLOod, Miss E. A. Univ. of Montana, Missoula, Mont.

§Flyns, B. D. Travelers Insurance Co., Hartford, Conn.

FlynN, J. D. Travelers Insurance Co., Hartford, Conn. $93 \mathrm{~N}$. Beacon St.

Focke, Dean T. M. Case School of Applied Science, Cleveland, Ohio.

Foley, J. G. Engineer, New York Central Railroad, New York, N. Y. $99353 d$ Ave., Brooklyn.

Foldey, Asst. Prof. K. W. Coll. of the City of Detroit, Detroit, Mich. 1216 E. Grand Blvd.

Foraker, Prof. F. A. Univ. of Pittsburgh, Pittsburgh, Pa. 1313 Macon Ave.

FORD, Asst. Prof. L. R. Rice Inst., Houston, Tex.

FORD, Prof. R. D. St. Lawrence Univ., Canton, N. Y. 58 Park St.

†FORD, Prof. W. B. Univ. of Michigan, Ann Arbor, Mich. 904 Forest Ave.

FORDER, Prof. H. G. Hymers Coll., Hull, England. 122 Goddard Ave.

ForsyTH, Asst. Prof. C. H. Dartmouth Coll., Hanover, N. H. Thayer Lodge.

ForT, Prof. Tomlinson. Lehigh Univ., Bethlehem, Pa.

$\S$ Fortescue, C. L. Consulting Transmission Engineer, Westinghouse Electric Co. E. Pittsburgh, $\mathrm{Pa}$.

Foster, Dr. A. L. Instr., Princeton Univ., Princeton, N. J. Prospect A pts.

Foster, Asso. Prof. M. C. Wesleyan Üniv., Middletown, Conn. 5 Long Lane.

Foster, R. M. Dept. Research, American Telephone and Telegraph Co., 195 Broadway, New York, N. Y.

Foster, Asst. Prof. W. I. Northern Montana School, Havre, Mont.

Fox, Pres. F. J. Fox Engineering Co., New York, N. Y. 1881 Walton Ave.

Fraleigh, Asst. Prof. P. A. Univ. of Vermont, Burlington, Vt. 109 Shelburne, Road.

Frank, D. H. George Washington High School, New York, N. Y. 3135 Decatur Ave., Bronx, N. Y.

Frankel, E. T. Statistician, National Industrial Conference Board, New York, N. Y. 295 Convent Ave.

Frankilin, Constance W. (Mrs. Phmip). 312 Pleasant St., Belmont, Mass

FrankliN, Dr. Fabian. Writer. 417 Riverside Drive, New York, N. Y.

Frankrin, Asso. Prof. Phrip. Massachusetts Inst. of Technology, Cambridge, Mass. 312 Pleasant St., Belmont, Mass.

Fréchet, Prof. Maurice. Institut Henri Poincaré, Univ. of Paris, Paris, France.

§FREDERICK, C. W. Eastman Kodak Co., Rochester, N. Y. 11 Fair View Heights.

FrEy, E. U. Structural Engineer. 4368 43d St., Merchantville, N. J.

FrINK, Asst. Prof. OrRIN. Pennsylvania State Coll., State College, Pa. 223 E. Nittany Ave.

Frisch, Prof. R. A. K. Visiting Prof., Yale Univ., New Haven, Conn. 100 Howe St.

Frumveller, Prof. A. F. Univ. of Detroit, Detroit, Mich. Livernois and 6 Mile Road.

FRy, Dr. T. C. Research Engineer, Bell Telephone Laboratories, 463 West St., New York, N. Y.

§Fuller, Prof. C. E. Massachusetts Inst. of Technology, Cambridge, Mass.

Fuller, Gordon. Instr., Univ. of New Mexico, Albuquerque, N. Mex.

FULLER, K. G. Edwardsport, Ind.

§Fulton, D. G. Univ. of Michigan, Ann Arbor, Mich. 333 E. William St.

GABA, Prof. M. G. Univ. of Nebraska, Lincoln, Neb. $1425 \mathrm{~S} 22 d \mathrm{St}$.

GAFAFER, W. M. Research Associate, Johns Hopkins Univ., Baltimore, Md. 185 Glenwood Ave., Leonia, N. $J$.

GAGE, Asst. Prof. W. H. Victoria Coll., Victoria, British Columbia, Canada.

Garnes, Prof. R. E. Univ. of Richmond, Richmond, Va.

GALE, Prof. A. S. Univ. of Rochester, Rochester, N. Y.

Gallatin, A. R. Smith and Gallatin, 66 Broadway, New York, N. Y.

Gallo, Prof. Joaquin. Director, National Astronomical Observatory, Tacubaya, Mexico. Zaragoza No. 127.

Garabedian, Asso. Prof. C. A. St. Stephens Coll. of Columbia Univ., Annandale-onHudson, N. Y. 
§Garabedian, H. A. John Hancock Mutual Life Insurance Co., 197 Clarendon Si., Boston, Mass.

Garabedian, Dr. H. L. Instr., Northwestern Univ., Evanston, Ill.

GarIs, Prof. C. F. F. Union Coll., Schenectady, N. Y.

Garretson, Asso. Prof. W. V. N. Oklahoma Agric. and Mech. Coll., Stillwater, Okla.

Garrett, Prof. W. H. Baker Univ., Baldwin City, Kans.

Garver, Asst. Prof. Raymond. Univ. of California at Los Angeles, Los Angeles, Calif. $1433 \mathrm{~W} .28 \mathrm{th}$ St.

Gavert, Asso. Prof. G. I. Univ. of Washington, Seattle, Wash. 5613 12th Ave., $N . E$.

Gay, Asst. Prof. H. J. Worcester Polytechnic Inst., Worcester, Mass.

Gehman, Prof. H. M. Univ. of Buffalo, Buffalo, N. Y. 67 Summit Ave.

GeORGES, Dr. J. S. Instr., Univ. of Chicago, Chicago, Ill.

Gergen, Dr. J. J. International Research Fellow. Harvard Univ., Cambridge, Mass.

Germond, H. H. C. F. Burgess Labs., 2103 Monroe St., Madison, Wis.

Gerst, Prof. F. J. St. Louis Univ., St. Louis, Mo.

Gibbens, Asst. Prof. Gladys E. C. Univ. of Minnesota, Minneapolis, Minn.

GIBBS, GEoRGE. Gibbs and Hill, Consulting Engineers, Pennsylvania Station, New York, N. Y.

Grison, Prof. I. L. Univ. of Utah, Salt Lake City, Utah.

Gilchrist, Asso. Prof. Lachlan. Univ. of Toronto, Toronto, Canada.

Grtl, Âsst. Prof. B. P. Coll. of the City of New York, New York, N. Y. 302 Convent Ave.

†GillespIe, Prof. D. C. Cornell Univ., Ithaca, N. Y. Cayuga Heights.

Gillessye, Prof. William. Princeton Univ., Princeton, N. J. Graduate Coll. Gilman, Ásst. Prof. R. E. Brown Univ., Providence, R. I. 44 E. Manning St.

GILMORE, R. O. Univ. of Wisconsin, Madison, Wis. North Hall.

GlasGow, Dr. JosepHiNe B. (Mrs.). 236 Manning Blvd., Albany, N. Y.

Glashan, Dr. J. S. C. 68 Somerset St., W. Ottawa, Canada.

Glazier, Asst. Prof. Harriet E. Univ. of California at Los Angeles, Los Angeles, Calif. 1307 Lucalle Ave.

Gleason, Prof. R. E. Temple Univ., Philadelphia, Pa. 2022 N. Park Ave.

GlenN, Prof. O.E. Univ. of Pennsylvania, Philadelphia, Pa. 127 McKinley Ave., Lansdowne, $P a$.

Glover, Pres. J. W. Teachers Insurance and Annuity Association of America, New York, N. Y. 522 Fifth Ave., Room 912.

§Goldobra, Barbara. Hunter Coll., Park Ave. and 68th St., New York, N. Y. 398 E. $152 d$ St.

GoldBerg, MiChaEL. Engineer. 623 19th St., N. W., Washington, D. C.

GOODMAN, JacoB. Instr., Rutgers Univ., New Brunswick, N. J.

\&Goodwin, Prof. H. M. Massachusetts Inst. of Technology, Cambridge, Mass.

Gordon, W. O. Instr., Pennsylvania State Coll., State College, Pa.

Gossard, Dean H. C. 'Nebraska Wesleyan Univ, Lincoln, Neb.

†Gould, ALICE B. Care of W. W. Vaughan, Exchange Bldg., Boston, Mass.

Gouris, Eli. Columbia Univ., New York, N. Y. 638 Crotona Park, S.

Gouwens, Asso. Prof. Cornelius. Iowa State Coll., Ames, Ia. 109 Hyland Ave.

Graber, Prof. M. E. Morningside Coll., Sioux City, Ia.

Graesser, Asst. Prof. R. F. Univ. of Arizona, Tucson, Ariz. Route 2, Box 158.

GraHaM, GEORGe. 3207 Washington Blyd., St. Louis, Mo.

Gratam, Asst. Prof. P. H. Washington Square Coll., New York Univ., New York, N. Y. 136 Brewster Ave., Flushing, N. Y.

Graham, W. J. Box 580, General Post Office, New York, N. Y.

Grant, AlICE A. Instr., Winthrop Coll., Rock Hill, S. C. Faculty House.

GraNT, H. S. Instr., Rutgers Univ., New Brunswick, N. J.

GRANT, J. D. Instr., Univ, of Michigan, Ann Arbor, Mich. 924 Forest Ave.

GRANville, Pres. W. A. U. S. National Life and Casualty Co., Chicago, Ill. 513 Aldine Ave., Lakeviere, Ill.

Graustein, Dr. Mary C. (Mrs. W. C.). Tutor, Radcliffe Coll., Cambridge, Mass. 32 Shepard St.

†Graustein, Asso. Prof. W. C. Harvard Univ., Cambridge, Mass. 32 Shepard St.

GravatT, Prof. T. E. Pennsylvania State Coll., State College, Pa. 500 E. College Ave.

Graves, C. H. Instr., Pennsylvania State Coll., State College, Pa. 225 S. Atherton St. 
Graves, Asso. Prof. G. H. Purdue Univ., Lafayette, Ind. 829 Mann St., W. Lafayette.

Graves, Asst. Prof. L. M. Univ. of Chicago, Chicago, Ill. Eckhart Hall.

Gray, Dr. Marion C. Dept. Research, American Telephone and Telegraph Co., 195 Broadway, New York, N. Y.

GREEN, Dr. C. F. Instr., Univ. of Illinois, Urbana, Ill. 803 W. Oregon St.

†Green, Prof. R. L. Stanford Univ., Stanford University, Calif.

Grennan, Dr. Elizabeth B. (Mrs. JoHN). 719 S. 7 th St., Ann Arbor, Mich.

Griffin, Prof. F. L. Reed Coll., Portland, Ore.

GrIfFITH, Asst. Prof. LoIs W. Northwestern Univ., Evanston, III.

†GroAT, B. F. Consulting Engineer. 20 Chapel St., Brookline, Mass

Gronwale, Dr. T. H. Consulting Mathematician. Care of Physics Dept., Columbia Univ., New York, N. Y. Physics Bldg

§Grout, H. A.' John Hancock Mutual Life Insurance Co., 197 Clarendon St., Boston, Mass.

Grove, Dr. C. C. Willoughby Branch, College of the City of New York, Brooklyn, N. Y. 143 Milbourn Ave., Baldwin, N. Y.

Grove, Asso. Prof. V. G. Michigan State Coll., East Lansing, Mich. 212 W. Grand River Ave.

Guggenbüh, Dr. Laura. Instr., Hunter Coll., Park Ave. and 68th St., New York, N. Y.

Gummer, Prof. C. F. Queen's Univ., Kingston, Ontario, Canada.

tGummere, Prof. H. V. Sevilla Court Apts., Bala-Cynwyd, Pa.

Gundersen, Prof. CARL. Oklahoma Agric. and Mech. Coll., Stillwater, Okla. 615 College Ave.

Gwinn, Asst. Prof. I. J. Morningside Coll., Sioux City, Ia.

Hackney, Prof. Lillian. Marshall Coll., Huntington, W. Va. 1230 11th St. HADLOCK, E. H. Instr., Cornell Univ., Ithaca, N. Y. 614 E. Buffalo St.

Hagen, Dr. Beatrice L. Ellinwood, Kans.

HAGLER, Capt. E. E. Adjutant General's Office, War Dept., Washington, D. C.

HaLberT, K. W. American Telephone and Telegraph Co., 195 Broadway, New York, N. $\dot{Y}$.

\$HALE, R. S. Edison Electric Illuminating Co., 39 Boylston St., Boston, Mass.

Haley, Prof. George. St. Ignatius Univ., San Francisco, Calif. 2241 Durant Ave., Berkeley, Calif.

Hall, Dr. DAN. Instr., Agric. and Mech. Coll., College Station, Texas.

HaLL, F. C. Instr., Rutgers Univ., New Brunswick, N. J. Apt. 9 B, 434 W. 120th St., New York, N. Y.

Hallett, Prof. G. H. Univ. of Pennsylvania, Philadelphia, Pa. College Hall. Halperin, Prof. Hilleil. Agric. amd Mech. Coll. of Texas, College Station, Tex. \$HAMIL ToN, H. C. Edison Electric Illuminating Co., 39 Boylston St., Boston, Mass. Hammatr, H. B. Instr., Lehigh Univ., Bethlehem, Pa.

Hammond, Prof. E. S. Bowdoin Coll., Brunswick, Me. 9 Thompson St.

Hampton, L. D. Instr., Alabama Polytechnic Inst., Auburn, Ala.

†Hanawalt, Prof. F. W. Coll. of Puget Sound, Tacoma, Wash. $826 \mathrm{~N}$. Steele St. HANCOCK, Prof. HARRIS. Univ, of Cincinnati, Cincinnati, Ohio. 2365 A uburn Ave.

\$ HanN, A. G. Pacific Mutual Life Insurance Co., Los Angeles, Calif.

$\$$ HanNa, C. R. Research Engineer, Westinghouse Electric Co., E. Pittsburgh, Pa. HanNa, Prof. U. S. Indiana Univ., Bloomington, Ind. 828 Atwater Ave.

Hannelly, Prof. R. J. Phoenix Junior Coll., Phoenix, Ariz.

Hanseld, S. B. Actuary, New York Telephone Co., 140 West St., New York, N. Y. Room 1308.

\$Haradon, R. S. Actuary, Aetna Life Insurance Co., Hartford, Conn.

$\$$ HARDCASTLE, E. E. Union Central Life Insurance Co., Cincinnati, Ohio. Box 892.

tHardC 4STLE, Frances. The Bramble Patch, Low Bridges, Stocksfield-on-Tyne, England.

HARDING, Prof. A. M. Univ. of Arkansas, Fayetteville, Ark.

HARDY, Prof. G. H. New Coll., Oxford, England.

†HARDY, Prof. J. G. Williams Coll., Williamstown, Mass.

HARGROVE, R. B. Glasgow, Ky.

Harkin, Asso. Prof. D. C. Alabama Polytechnic Inst., Auburn, Ala. Box 214.

Harmovir, G. P. East High School, Columbus, Ohio. 2290 Indianola Ave.

\$HARMS, D. H. Turbine Engineering Dept., General Electric Co., Schenectady, N. Y. 
Harper, F. S. Instr., Univ. of Nebraska, Lincoln, Neb. $306 M$. A., Station A. Harris, Elizabeth L. Southwestern Bell Telephone Co., St. Louis, Mo. $336 W$. Madison St., Kirkwood, Mo.

Harshbarger, Prof. W. A. Washburn Coll., Topeka, Kans. 1401 College Ave.

HarT, Prof. W. L. Univ. of Minnesota, Minneapolis, Minn. Room 119, Folwell Hall.

Hart, Asst. Prof. W. W. Univ, of Wisconsin, Madison, Wis.

HARTER, Prof. G. A. Univ. of Delaware, Newark, Del. 2014 Delaware Ave., Wilmington, Del.

§HARTER, IsAAC. Babcock and Wilcox Co., 85 Liberty St., New York, N. Y.

Hartley, Prof. R. W. Southwestern Univ., Memphis, Tenn.

Hartmann, Prof. F. M. Cooper Union, New York, N. Y.

Hartung, M. L. Instr., Univ. of Wisconsin, Madison, Wis. North Hall.

Hartwel, George. Cheltenham Magnetic Observatory, Cheltenham, Md.

HarTwick, Prof. F. C. Univ. of Dayton, Dayton, Ohio.

Haseman, Prof. Charles. Univ. of Nevada, Reno, Nev.

Haseman, Prof. Mary G. Hartwick Coll., Oneonta, N. Y.

HAskell, Prof. M. W. Univ. of California, Berkeley, Calif. Box 3.

Haskins, Prof. C. N. Dartmouth Coll., Hanover, N. H. Hanover Road, Lebanon, N. $H$

Hassler, Prof. J. O. Univ. of Oklahoma, Norman, Okla. 425 Lahoma Ave.

Hatch, Asso. Prof. D. A. Lafayette Coll., Easton, Pa. 705 High St.

Hathaway, Prof. A. S. Boerne, Tex.

HaUsie, Eugenie C. 1331 Chisholm St., Bronx, New York, N. Y.

§Havens, A. W. Pacific Mutual Life Insurance Co., Los Angeles, Calif.

Haviland, Asst. Prof. E. K. Lincoln Univ., Chester County, Pa Port Deposit, Md.

Hawkes, Dean H. E. Columbia Univ., New York, N. Y.

Hawkins, ERNEst. Instr., U. S. Naval Academy, Ännapolis, Md. 39 Murray Ave

Hawks, Lena J. Ward-Belmont Coll., Nashville, Tenn.

HAyashi, Prof. TsuruTCHI. Coll. of Science, Tôhoku Imperial Univ., Sendai, Japan.

Hayden, Camilla. $311 \mathrm{~N}$. Charter St., Madison, Wis.

Haynes, Prof. E. S. Univ. of Missouri, Columbia, Mo. 206 Physics.

HAZARD, Asst. Prof. W. J. Univ, of Colorado, Boulder, Colo. 1001 10th St.

Hazeltine, L A. Stevens Inst. of Technology, Hoboken, $\mathbf{N}$ J.

tHazlett, Asst. Prof. Olrve C. Univ. of Illinois, Urbana, III.

H'Doubler, Dr F. T. Surgeon, Medical Arts Bldg., Springfield, Mo.

HebBERT, Dr. C. M. Engineer, Bell Telephone Laboratories, 463 West St., New York, N. Y.

\$Heckel, MAR JORIE L. Brown Univ., Providence, R. I.

Hedudnd, Dr. G. A. Associate, Bryn Mawr Coll., Bryn Mawr, Pa.

HEDrick, Prof. E. R. Univ. of California at Los Angeles, Los Angeles, Calif.

HEDRICK, Dr. H. B. Ballistician, Office of the Chief of Ordnance, Washington, D. C. 3240 S St.

\$Hermberger, G. J. Babcock and Wilcox Co., Bayonne, N. J. 46 Ave. $B$.

Heineman, Asst. Prof. E. R. Texas Technological Coll., Lubbock, Tex. $232 W$. Lake St., Lake Mills, Wis.

Heldiweld, C. H. Instr., New York Univ., New York, N. Y. 100 Washington Square E.

Heminger, I. N. 84 S. First St., San José, Calif.

Hempstead, J. C. Instr., Drexel Inst., Philadelphia, Pa. 32d and Chestnut Sts.

Henderson, Prof. ARChibald. Univ. of North Carolina, Chapel Hill, N. C.

§HENDERSON, M. L. Engineering General Dept., General Electric Co., Schenectady, N. Y.

Henderson, Robert. Vice-Pres., Equitable Life Assurance Society, 393 7th Ave, New York, N. Y.

Hennel, Asso. Prof. CoRA B. Indiana Univ., Bloomington, Ind. 822 Atwater Ave.

HEROD, W. R. Care of International General Electric Co., 120 Broadway, New York, N. Y.

Herr, Asso. Prof. Gertrude A. Iowa State Coll., Ames, Ia. Cranford Apt. 30.

Hess, Asso. Prof. G. W. Howard Coll., Birmingham, Ala. 8009 Berney Ave.

Hestenes, M. R. Univ. of Chicago, Chicago, Ill. Hitchcock Hall.

Hewes, Dr. L. I. U. S. Bureau of Public Roads, San Francisco, Calif, Sheldon Bldg. 
Hickey, Dr. Deborah M. 511 Harvard St., Houston, Texas.

HICks, Prof. H. C. Texas Technological Coll., Lubbock, Tex.

HICKson, Asst. Prof. A. O. Duke Univ., Durham, N. C.

Hightower, Prof. Ruby U. Shorter Coll., Rome, Ga.

\$HILDEBRANDT, E. H. Univ. of Michigan, Ann'Arbor, Mich. 1721 Wells $s t$

Hm.derrandT, Prof. T. H. Univ. of Michigan, Ann Arbor, Mich. 1930 Cambridge Road.

\$Hill, E. B. Aetna Life Insurance Co., Hartford, Conn.

HuL, Asso. Prof. L. S. Hunter Coll., Park Ave. and 68th St., New York, N. Y. 1180 Anderson Ave., Bronx.

Hillard, Asso. Prof. C. R. Wheaton Coll., Wheaton, IH.

Hille, Asso. Prof. Einar. Princeton Univ., Princeton, N. J. 174 Prospect Ave.

HuLLs, E. J. Instr., Los Angeles Junior Coli., Claremont, Calif. $125 \mathrm{~W}$. 11th St.

HiLtebertel, Dr. A. M. Trappe, Montgomery County, Pa.

Hilton, Prof. Harold. Bedford Coll., Univ of London, Regents Park, London, N. W. 1, England.

Himwich, Dr. A. A. 1871 Madison Ave., New York, N. Y.

Hinrichsen, Asst. Prof. J. J. L. lowa State Coll., Ames, Ia.

Hinsch, Asso. Prof. V. B. Missouri School of Mines, Rolla, Mo. 1106 Rolla St.

Hirayama, Arira. Yamagata-Women's Normal School, Narita-Machi, Chiba ken, Japan.

Hirschler, Prof. E. J. Bluffton Coll, Bluffton, Ohio. W. Elm St.

Hitchcock, Prof. R. R. Univ. of North Dakota, Grand Forks, N. Dak.

Hobart, Prof. C. M. Teachers Coll., Aberdeen, S. Dak.

Hodge, Asst. Prof. F. H. Purdue Üniv., Lafayette, Ind. $820 \mathrm{~N}$. Main St., W. Lafayette.

Hodgkins, Dean H. L. George Washington Univ., Washington, D. C.

§Hoel, P. G. Univ. of Minnesota, Minneapolis, Minn. 2212 Grand Ave.

HoErsch, Dr. V. A. Instr., Univ. of Illinois, Urbana, Ill. 909 S. First St., Champaign, Ill.

HofmanN, Dr. LuLu. Instr., Columbia Univ., New York, N. Y. 88 Morningside Drive.

§НоваUS, R. A. Asst. Actuary, Metropolitan Life Insurance Co., 1 Madison Ave., New York, N. Y.

Holden, A. N. Technical Publicity, Bell Telephone Laboratories, 463 West St, New York, N. Y.

Holder, Prof. F. J. Mercer Univ., Macon, Ga. 912 College St.

Holgate, Prof. T. F. Northwestern Univ., Evanston, Ill. 617 Library Place.

Holl, Asso. Prof. D. L. Iowa State Coll., Ames, Ia. 2323 Donald St.

HoltCROFT, Prof. T. R. Wells Coll., Aurora, N. Y.

HolleY, Dr. J. L. Investment Research. 8 Irvington St., Boston, Mass.

Holmes, Asst. Prof. C. T. Bowdoin Coll., Brunswick, Me.

Holmes, Helma L. Univ. of Texas, Austin, Tex. 1908 Unversity Ave.

Hood, Catherine. Instr., Colorado Coll., Colorado Springs, Colo.

Hoover, Asst. Prof. B. P.' Carnegie Inst. of Technology, Pittsburgh, Pa.

Hoover, H. A. Instr., Univ. of West Virginia, Morgantown, W. Va.

Hopkins, Dr. Charles. Univ, of Illinois, Urbana, Ill. 359 Math. Bldg.

Hopkins, FanNIE. 234 E. Park Ave., Waukesha, Wis.

Hopkins, Asso. Prof. L. A. Univ. of Michigan, Ann Arbor, Mich. 1517 S. Unversity Ave.

Hornberger, H. J. Great Northern Life Insurance Co., Chicago, Ill. $110 \mathrm{~S}$. Dearborn St.

Horne, Prof. C. E. University of Porto Rico, Santurce Station, San Juan, Porto Rico. Box 3024.

Horsfall, I. O. Instr., Cornell Univ., Ithaca, N. Y. 119 Blair St.

Horton, Adj. Prof. Goldie P. Univ. of Texas, Austin, Tex. $504 \mathrm{~W}$. 32d St.

Horsford, Prof. H. M. Univ. of Arkansas, Fayetteville, Ark.

§Hoskins, J. E. Asst. Actuary, Travelers Life Insurance Co., Hartford, Conn. Hoskins, Prof. L. M. Stanford Univ., Stanford University, Calif.

§HOSMER, SidNEy. Edison Electric Illuminating Co., 39 Boylston St., Boston, Mass.

Hotelling, Asso. Prof. Harold. Stanford Univ., Stanford University, Calif.

HouseHOLDER, A. S Instr., Washburn Coll, Topeka, Kans.

§HovgAaRd, Prof. WILliam. Massachusetts Inst. of Technology, Cambridge, Mass.

§Howard, A. Engineering General Dept., General Electric Co., Schenectady, N. Y. 
Howe, Asst. Prof. Anna M. Newcomb Coll., New Orleans, La.

\$Howe, L. H. John Hancock Mutual Life Insurance Co., 197 Clarendon St., Boston, Mass.

\$Howell, VAlENTINe. Prudential Insurance Co., Newark, N. J.

Howland, Prof. L. A. Wesleyan Univ., Middletown, Conn.

Hoyt, R. S. Telephone Research Engineer, American Telephone and Telegraph Co., 195 Broadway, New York, N. Y.

Hubss, Asst. Prof. H. N. Hobart Coll., Geneva, N. Y. 87 Madison St.

Huber, Prof. C. M. Atlantic Univ., Virginia Beach, Va.

Hubert, Asst. Prof. W. G. Coll. of the City of New York, New York, N. Y.

\$Hugres, Dean H. J. Engineering School, Harvard Univ., Cambridge, Mass. 223 Pierce Hall.

Hughes, Asso. Prof. Jewell C. Univ. of Arkansas, Fayetteville, Ark.

Hughes, Olive M. Fellow, Bryn Mawr Coll., Bryn Mawr, Pa.

Huke, Dr. Aline Instr., Pennsylvania State Coll , State College, Pa.

Hult, Prof. Daniel. Univ. of Notre Dame, Notre Dame, Ind.

Hume, Prof. AlFred. Univ. of Mississippi, University, Miss.

Humpureys, Prof. W. J. U. S. Weather Bureau, Washington, D. C. Cosmos Club. HuNdLey, Asso. Prof. R. E. Univ. of Cincinnati, Cincinnati, Ohio.

Hunt, Asst. Prof: G. H. Univ. of California at Los Angeles, Los Angeles, Calif.

Hunt, Asst. Prof. Mildred. Illinois Wesleyan Univ., Bloomington, Ill. $1211 \mathrm{Fell}$ Ave.

\$Hunter, R. G. Equitable Life Insurance Co. of Iowa, Des Moines, Ia.

tHuntrongton, Prof. E. V. Harvard Univ., Cambridge, Mass. 48 Highland St. \$Huntley, H. B. Instr., Rutgers Coll., New Brunswick, N. J.

Hurry, Asst. Prof. J. A. Western State Coll., Gunnison, Colo.

HuRst, Asso. Prof. J. W. Montana State Coll., Bozeman, Mont

†Hurwitz, Prof. W. A. Cornell Univ., Ithaca, N. Y. 8 Whate Hall.

Hussey, L. W. Electrical Engineer, Bell Telephone Laboratories, 463 West St., New York, N. Y.

Hutcheson, W. A. Mutual Life Insurance Co., 34 Nassau St., New York, N. Y.

Hutchinson, Prof. C. A. Univ of Colorado, Boulder, Colo.

HutChinson, Prof. J. I. Cornell Univ., Ithaca, N. Y.' 140 Thurston Ave.

§Hyde, Asso. Prof. Emma. State Agric. Coll., Manhattan, Kans.

Ickes, E. L. Geologist. 10463 Tennessee Ave., Los Angeles, Calif.

IILICK, J. E. Instr., Lehigh Univ., Bethlehem, Pa.

INCE, Prof. E. L. Egyptian Univ., Cairo, Egypt.

Ingoud, Prof. Byron. Culver-Stockton Coll., Canton, Mo.

Ingold, Asst. Prof. Louss. Univ. of Missouri, Columbia, Mo. 8 Hamilton Way. Ingraham, Prof. M. H. Univ. of Wisconsin, Madison, Wis. 110 Virginia Terrace INGRaM, W. H. Univ, of Washington, Seattle, Wash. Philosophy Hall.

InwIN, Asso. Prof. Frank. Univ. of California, Berkeley, Calif. 2921 Regent St. \$IRwiN, P. C. Equitable Life Insurance Co. of Iowa, Des Moines, Ia.

IsaAcs, Prof. C. A. State Coll. of Washington, Pullman, Wash.

†Jackson, Prof. Dunham. Univ. of Minnesota, Minneapolis, Minn. 119 Folwell Hall.

JAckson, Asso. Prof. J. B. Univ. of South Carolina, Columbia, S. C. 227 S. Waccamaw Ave.

JACKson, Dr. L. L. First Asst. Superintendent of Schools, Newark, N. J.

JACKson, Dr. Rosa L. Instr., Hunter Coll., Park Ave. and 68th St., New York, N. Y. 2705 Marron Ave.

JAcoBUS, Dr D. S. Babcock and Wilcox Co., 85 Liberty St., New York, N. Y. JAEGER, Asst. Prof. C. G. Tulane Univ., New Orleans, La.

JaIME, Dr. F. D. Instituto Nacional Profesorado, Buenos Aires, Argentina. Concordia 196

James, Asso. Prof. Glenn. Univ. of California at Los Angeles, Los Angeles, Calif.

JAMEs, Prof. G. O. Washington Univ., St. Louis, Mo.

James, Prof. Vern. Menlo Junior Coll., Menlo, Calif. 604 Tennyson Ave, Ralo Alto, Calnf.

Janes, Asst. Prof. W. C. State Agric. Coll., Manhattan, Kans,

Jefrery, Prof, R. L. Acadia Univ., Wolfville, Nova Scotia.

\$JEKEL, A. H. Mountain Plains Co., Inc., Boulder, Colo. Box 182.

JENSEN, Dr. C. M. 3315 17th Ave., S., Minneapolis, Minn. 
JERBERT, Dr. A. R. Instr., Univ. of Washington, Seattle, Wash.

†JoFfe, S. A. Mutual Life Insurance Co., 32 Nassau St., New York, N. Y.

JoHN, Asst. Prof. F. W. New York Univ., New York, N. Y. Washington Sq. E.

§ounson, E. E. Engineering General Dept., General Electric Co., Schenectady, N. Y.

Johnson, E. H. Instr., New York Univ., University Heights, New York, N. Y.

Johnson, Prof. E. N. Butler Univ., Indianapolis, Ind. 304 Downey Ave.

JoHnson, H. A., JR. 2275 Chevy Chase, Glendale, Calif

Jornson, Asst. Prof. MarIe M. Oberlin Coll., Oberlın, Ohio. $116 \mathrm{Elm} \mathrm{St}$.

Johnson, Prof. Myra I. Whitney Women's Coll., New York, N. Y. 38, 430 West 120th St.

Johnson, Asso. Prof. R. A. Brooklyn Coll. of the City of New York, Brooklyn, N. Y. 66 Court St.

Johnson, Asso. Prof. R. P. Carnegie Inst. of Technology, Pittsburgh, Pa.

Johnson, W. C. Research Laboratory, General Electric Co., Schenectady, N. Y.

Jornston, Asst. Prof. F. E. George Washington Univ., Washington, D. C. 815 Connecticut Ave.

Johnston, Prof. L. S. Univ. of Detroit, Detroit, Mich.

Jonah, Asst. Prof. F. C. Adelbert Coll., Western Reserve Univ., Cleveland, Ohio.

§JoNAH, H. F. S. Instr., Purdue Univ., Lafayette, Ind.

Jones, Asst. Prof. B. W. Cornell Univ., Ithaca, N. Y.

Jones, Prof. E. H. Southern Methodist Univ., Dallas, Tex.

Jones, Prof. J. L. Akron Univ., Akron, Ohio. 49 Kathron Ave., Cuyahoga Falls, Ohio.

§ones, L. A. Eastman Kodak Co., Rochester, N. Y. 157 Birr St.

JoNes, P. C. Bell Telephone Laboratories, New York, N. Y. Bureau of Publications.

§Jones, W. A. Babcock and Wilcox Co., Bayonne, N. J.

Jordan, Prof. Charles. Univ. of Budapest, Budapest, Hungary, 1 Berenyi-ut 7.

Jordan, Asso. Prof. H. E. Univ. of Kansas, Lawrence, Kans. 1600 Kentucky St.

Kaplan, Dr. H. G. Stuyvesant High School, 15th St. and First Ave, New York, N. Y. 1403 Nelson Ave., Bronx.

†Karapetoff, Prof. Vladimir. Cornell Univ., Ithaca, N. Y. 520 E. Buffalo St.

\$Karelitz, G. R. Research Engineer, Westinghouse Electric Co., East Pittsburgh, $\mathrm{Pa}$.

Karnow, Herman. Brown Univ., Providence, R. I.

Karpinski, Prof. L. C. Univ. of Michigan, Ann Arbor, Mich. 1315 Cambridge Road.

†Kasner, Prof. Edward. Columbia Univ., New York, N. Y. 22 W. 119th St.

KawaguchI, AkITsugu. Lecturer, Coll. of Science, Hokkaido Imperial Univ., Sapporo, Japan. Care of Prof. Takasu, Tôhoku Imperal Unvv., Sendar, Japan.

KazarinoFF, D. C. Instr., Univ. of Michigan, Ann Arbor, Mich. 1515 Cambrudge Road.

Kearney, Prof. Dora E. Iowa State Teachers Coll., Cedar Falls, Ia. 1217 W. 23d St.

KEE, OLIVE A. Teachers Coll., Huntington, Ave., Boston, Mass.

KeELeR, C. A. Instr., Lehigh Univ., Bethlehem, Pa. 528 Fourth Ave.

Keesing, Maurice. 1549 Leland Ave., New York, N. Y.

Keffer, RalpH. Asst. Actuary, Aetna Life Insurance Co., Hartford, Conn.

Keller, Dr. E. G. General Electric Co., Schenectady, N. Y.

KellogG, Prof. O. D. Harvard Univ., Cambridge, Mass. $18 \mathrm{Crang} e \mathrm{St}$.

Kells, Asst. Prof. L. M. U. S. Naval Academy, Annapolis, Md. 312 West St.

KempNer, Prof. A. J. Univ. of Colorado, Boulder, Colo.

Kendall, Asso. Prof. Claribel. Univ. of Colorado, Boulder, Colo. 1305 Euchd Ave.

Kennedy, Capt. G. S. Ordnance Dept., U. S. Army. Raritan Arsenal, Metuchen, N. J.

Kennelly, Prof. A. E. Harvard Univ., Cambridge, Mass.

Kennison, Dr. L. S. Research Asst., Princeton Univ., Princeton, N. J.

KenNy, MARY V. Instr., Hunter Coll., Park Ave. and 68th St., New York, N. Y. DE KerékJÁrtó, BeLA. 'Univ. of Szeged, Szeged, Hungary.

Kern, Dorothea A. Instr., Cascadilla Prep. School, 444 Kenwood Road, Drexel Park, Delaware County, Pa.

\$KerR, H. J. Babcock and Wilcox Co., 85 Liberty St., New York, N. Y. 639 Chestnut St, Westield, N.J. 
Kersten, H. J. Instr., Univ. of Cincinnati, Cincinnati, Ohio.

Ketchum, Dr. P W Associate, Univ. of Illinois, Urbana, Ill. 363 Math. Bldg.

\$KEYes, Prof. F. G. Director of Research Laboratory of Physical Chemistry, Massachusetts Inst. of Technology, Cambridge, Mass.

tKeyser, Prof. C. J. Columbia Univ., New York, N. Y.

Kexser, Sarah Y. (Mrs. C. J.). 302 Convent Ave., New York, N. Y.

Krolodovsky, E. A. Columbia Univ., New York, N. Y. 16 E. 129th St.

Kiang, Dr. Tsar-Hau. Research Asst., Princeton Univ., Princeton, $\mathrm{N}$ J.

Kichline, W. L. Instr., Lehigh Univ., Bethlehem, Pa. 400 Sixteenth Ave.

Kullebrew, Prof. C. D. Alabama Polytechnic Inst., Auburn, Ala.

\$KimbatL, A. L. Research Laboratory, General Electric Co., Schenectady, N. Y.

Krmball, Asso. Prof. B. F. Univ. of New Hampshire, Durham, N. H.

KimbalL, Prof. R. S. Superintendent of Schools, N. Brookfield, Mass.

Kimball, S. H. 67 Goden St., Belmont, Mass.

Kimball, Asst. Prof. W. S. Michigan State Coll., East Lansing, Mich. Campus $A$ pis.

Kindle, Prof. J. H. Univ. of Cincinnati, Cincinnati, Ohio.

§KINEKE, F. D. Prudential Insurance Co., Newark, N. J.

King, Dr. Eula W. (Mrs. H. L.). 3228 Lafayette Ave., St. Louis, Mo.

KInG, Prof. L. V. McGill Univ., Montreal, Canada.

Kingston, Prof. H. R. Univ. of Western Ontario, London, Ontario, Canada.

Kinney, Dr. J. M. Crane Junior Coll., Chicago, Ill. 8058 Bennett Ave.

Kinsman, A. D. 80 County Road, Ipswich, Mass.

KIRCHER, Dr. E. A. T. 808 Woodland Ave., Plainfield, N. J.

KLAUber, L. M. Consolidated Gas and Electric Co., San Diego, Calif. $233 \mathrm{~W}$. Juniper St.

KLEIN, A. L. California Inst. of Technology, Pasadena, Calif.

KLINE, Prof. J. R. Univ. of Pennsylvania, Philadelphia, Pa. Riverview Ave., Swarthmore, $P a$.

KLINE, Rose. $226-8$ E. Fourteenth St., New York, N. Y.

Knebelman, Asst. Prof. M. S. Princeton Univ., Princeton, N. J.

Knedler, P. A. Instr., Univ. of Pennsylvania, Philadelphia, Pa. College Hall.

KNIGHT, Asst. Prof. ElizabeTh E. Milwaukee State Normal School, Milwaukee, Wis. 627 Downer Ave.

KNISEly, Alexander. Columbia City, Ind.

KNOBELAUCH, E. A. Instr., Long Island Univ., Brooklyn, N. Y.

KoEHLER, T. L. Instr., Muhlenberg Coll., Allentown, Pa. 625 N. 24th St.

\$Koeppe, A. J. Union Central Life Insurance Co., Cincinnati, Ohio. Box 892.

KoKomoor, Asso. Prof. F. W. Univ. of Florida, Gainesville, Fla.

Konantz, Prof. Emma L. Yen Ching Univ., Peking, China.

Koopman, Asst. Prof. B. O. Columbia Univ., New York, N. Y.

§KopF, E. W. Metropolitan Life Insurance Co., 1 Madison Ave., New York, N. Y.

Kormes, $J$. $\dot{P}_{\text {. }}$ (Mrs. MARK). Instr., Hunter Coll., Park Ave. and 68th St., New York, N. Y. 615 Ocean Ave., Brooklyn, N. Y.

KoRmES, Dr. MARK. 615 Ocean Ave., Brooklyn, N. Y.

Korn, Prof. ARTHUR. Technical School, Berlin-Charlottenburg, Germany. Schluterstr. 25.

KorzyBSkI, AlFred. Engineer. Fifth Ave. Bank, New York, N. Y.

Kovalenko, Asst. Prof. Michael. Swarthmore Coll., Swarthmore, Pa.

KovariK, Prof. A. F. Yale Univ., New Haven, Conn. Sloane Physics Laboratory.

KRAFT, ONA. East High School, E. 83d St., Cleveland, Ohio. 14174 Superior Road, Heighs Branch.

§Krali, H. L. Brown Univ., Providence, R. I.

Kramer, Asst. Prof. EdNa E. New Jersey State Teachers Coll., Montclair, N. J. 1520 Carroll St, Brooklyn.

KrathwoHL, Asso. Prof. W. C. Armour Inst. of Technology, 33d and Federal Sts., Chicago, IIl.

Kubota, Prof.'T. Tôhoku Imperial Univ., Sendai, Japan.

Kunn, Prof. H. W. Ohio State Univ., Columbus, Ohio. 1145 Fairverw Ave., Grandvew Heights.

Kuniyeda, Prof. MotojI. Tokyo Higher Normal Coll., Tokyo, Japan.

KunTe, HeleN. Instr., Hunter Coll., Park Ave. and 68th St., New York, N. Y.

KuRzIN, W. H. Crane Junior Coll., Chicago, Ill. 1631 Roosevelt Road.

†Kuschke, Prof. C. G. P. Univ. of Porto Rico, Rio Piedras, Porto Rico.

KusNer, Asst. Prof. J. H. Univ. of Florida, Gainesville, Fla. 
†Ladue, Pomeroy. Public Accountant. 683 Prentis Ave., Detroit, Mich.

LAmbeRT, W. D. U. S. Coast and Geodetic Survey, Washington, D. C.

Lamond, Dr. J. K. Telephone Engineer. 4 Pennsylvania Ave., Brookline, Delaware County, $\mathrm{Pa}$.

Lampland, C. O. Lowell Observatory, Flagstaff, Ariz.

Lamson, Asst. Prof. K. W. Lehigh Univ., Bethlehem, Pa.

LANDERS, A. W., JR. Instr., Brooklyn Coll of the City of New York, Brooklyn, N. Y.

LANDIS, Prof. W. W. Dickinson Coll., Carlisle, Pa.

LANDRY, Prof. A. E. Catholic Univ., Washington, D. C. 3624 13th St., Brookland, D. C.

Lane, Prof. E. P. Univ. of Chicago, Chicago, Ill

LANE, Asso. Prof. H. I. Univ. of South Dakota, Vermilion, S. Dak.

Langdon, W. H. Yale Univ., New Haven, Conn. 63 Gillett St., Hartford, Conn.

LANGer, Prof. R. E. Univ. of Wisconsin, Madison, Wis. 521 S. Randall Ave.

Langrord, Asso. Prof. C. H. Univ. of Michigan, Ann Arbor, Mich. Dept. of Philosophy.

Langman, Dr. Harry. Manufacturing Chemist. 6919 Benchell St., Arverne, New York, N. Y.

§LangmuiR, Dr. I. Research Laboratory, General Electric Co., Schenectady, N. Y.

\$LANGVAND, L. L. Babcock and Wilcox Co., Barberton, Ohio.

LaPaz, Asst. Prof. Lincoln. Ohio State Univ., Columbus, Ohio.

LAREW, Prof. GILIIE A. Randolph-Macon Woman's Coll., Lynchburg, Va.

LARMOR, Sir JoSePH. St. John's Coll., Cambridge, England.

LARSEN, H. D. Instr., Univ. of Wisconsin, Madison, Wis.

LARUS, J. R. Phoenix Mutual Life Insurance Co., Hartford, Conn.

LASLEX, Prof. J. W. Univ. of North Carolina, Chapel Hill, N. C. 523 E. Rosemary Lane.

LATrmer, Prof. C. G. Univ. of Kentucky, Lexington, Ky.

LavN, D. D. Sheridan Hotel, Minneapolis, Minn.

Laves, Asso. Prof. Kurt. Úniv, of Chicago, Chicago, Ill. 5611 Kenwood Ave.

Leavens, D. H. Research Staff, Harvard Business School, Soldier's Field, Boston, Mass.

Lefschetz, Prof. Sotomon. Princeton Univ., Princeton, N. J. 190 Prospect St.

Lehmer, D. H. National Research Fellow, California Inst. of Technology, Pasadeñ, Calif.

Lehmer, Prof. D. N. Univ. of California, Berkeley, Calif. 2736 Regent St.

Lehr, Dr. A. MARguerite M. Associate, Bryn Mawr Coll., Bryn Mawr, Pa. Low Buildings.

LerB, Prof. D. D. Connecticut Coll, for Women, New London, Conn. 358 Mohegan Ave.

§LEIbING, J. K. General Engineering Laboratory, General Electric Co., Schenectady, N. Y.

Leinbach, ERMa M. 317 N. Fifth St., Reading, Pa.

tLennes, Prof. N. J. Univ. of Montana, Missoula, Mont.

Lenzen, Asso. Prof. V. F. Univ. of California, Berkeley, Calif. Physics Dept.

Leonard, Prof. H. B. Univ. of Arizona, Tucson, Ariz.

LEPESHKIN, S. A. Instr., Worcester Polytechnic Inst., Worcester, Mass.

Lester, Prof. O. C. Univ. of Colorado, Boulder, Colo. 1061 11th St.

LeStourgeon, Asso. Prof. Flora E. Univ. of Kentucky, Lexington, Ky. 630 Maxwelton Court.

Leuschner, Prof. A. O. Director, Students' Observatory, Univ. of California, Berkeley, Calif. 1816 Scenic Ave.

Levi-Civita, Prof. Tullio. Univ. of Rome, Rome, Italy. Via Sardegna 50.

LEVINE, JACK. Univ. of California at Los Angeles, Los Angeles, Calif.

Levinson, Dr. H. C. 4049 Lake Park Ave., Chicago, Ill.

Levy, Asst. Prof. Harry. Univ. of Illinois, Urbana, Ill.

Levy, Asst. Prof. Sophia H. Univ. of California, Berkeley, Calif. 453 Wheeler Hall.

Lewandowski, StepHen. Marquette Unıv, Milwaukee, Wis.

Lewis, Prof. Anna D. Lake Erie Coll., Painesville, Ohio. 38 Paige Lane.

Lewrs, Asst. Prof. A. J. Univ. of Denver, Denver, Colo. 2112 S. Columbine St.

LEwIs, D. C. Instr., Lehigh Univ., Bethlehem, Pa.

Lews, Asst. Prof. F. A. Univ. of Alabama, University, Ala. Box 365.

Lewis, Prof. Florence P. Goucher Coll., Baltimore, Md.

\$LEWIS, N. E. Babcock and Wilcox Co., 85 Liberty St., New York, N. Y.

Libman, Dr. E. E. Engineer, Aeronautics and Marine Engineering Dept., General Electric Co., River Road, Schenectady, N. Y. 
LICHTER, J. J. Consulting Engineer 6840 Waterman Ave., St. Louis, Mo.

Lieber, H. G. Columbia Univ., New York, N. Y. 208 Washington Park, Brooklyn.

Lindsay, Asso. Prof. R. B. Brown Univ., Providence, R. I. Physics Dept.

LindSEY, Prof. Lours. Syracuse Univ., Syracuse, N. Y. 201 Scottholm Blvd.

Linehan, Prof. P. H. Coll. of the City of New York, New York, N. Y. 346 Convent Ave.

Linfield, Asso. Prof. B. Z. Univ. of Virginia, University, Va. Faculty Apts.

LING, Prof. G. H. Univ. of Saskatchewan, Saskatoon, Canada.

Linker, Asso. Prof. J. B. Univ. of North Carolina, Chapel Hill, N. C.

Linton, M. A. Provident Mutual Life Insurance Co., 409 Chestnut St., Philadelphia, $\mathrm{Pa}$.

LirtaUter, S. B. National Research Fellow. Harvard Univ., Cambridge, Mass.

Litzinger, Asst. Prof. Marie. Mount Holyoke Coll., South Hadley, Mass. Porter Hall.

Liv, Dr. Chin-Nien. Instr., Nankai Univ., Tientsin, China.

Livens, Prof. G. H. University Coll., Cardiff, Wales.

LOCKE, L. L. 950 St. John's Place, Brooklyn, N. Y.

Logsdon, Asst. Prof. Mayme I. (Mrs.). Univ. of Chicago, Chicago, Ill.

LONG, Asst. Prof. T. R. Univ. of Rochester, Rochester, N. Y. 75 Cypress St.

LONGENECKER, J. V. S. Farmers and Bankers Life Insurance Co., 701 Beacon Bldg., Wichita, Kans.

Longley, Prof. W. R. Yale Univ., New Haven, Conn. 595 Orange St.

§LorraINE, R. G. Engineering General Dept., General Electric Co., Schenectady, N. Y.

de Losada y Puga, Dr. Cristobal. Apartado 2708, Lima, Peru.

LotKA, Dr. A. J. Supervisor of Mathematical Research, Metropolitan Life Insurance Co., 1 Madison Ave., New York, N. Y.

Love, Prof. A. E. H. Oxford Univ., Oxford, England. 34 St. Margaret's Road.

Love, Prof. C. E. Univ of Michigan, Ann Arbor, Mich.

Lovel, , C. A. Bell Telephone Laboratories, 463 West St., New York, N. Y. 640 Fort Washington Ave.

tLovetT, Pres. E. O. Rice Inst., Houston, Tex.

LowENsTern, L. L. Instr., Cornell Univ., Ithaca, N. Y. White Hall.

\$LowneY, R. E. Instr., Univ. of Wisconsin, Madison, Wis. North Hall.

Lubben, Adj. Prof. R. G. Univ. of Texas, Austin, Tex. $708 \mathrm{~W} .221 / 2 \mathrm{St}$.

Lubin, Asst. Prof. C. I. Univ. of Cincinnati, Cincinnati, Ohio. College of Engineering.

Luby, Prof. W. A. Kansas City Junior Coll., Kansas City, Mo. 5411 Rockhill Road.

Luck, Prof. J. J. Univ. of Virginia, University, Va. Box 214.

\$Lundaren, E. A. Prudential Insurance Co., Newark, N. J.

Lunv, Prof. A. C. Univ. of Chicago, Chicago, Ill. 5211 Kenwood Ave.

Lusk, Prof. H. F. Sacramento Junior Coll., Sacramento, Calif.

LUTHER, C. F. Instr., Leland Stanford Univ., Stanford University, Calif. Box 2714.

Lyman, Prof. E. A. State Normal School, Ypsilanti, Mich. $126 \mathrm{~N}$. Washington St.

\$Lyman, Prof. Theodore. Harvard Univ., Cambridge, Mass.

LynCH, Prof. D. J. "Boston Coll., Chestnut Hill, Mass.

LytLe, Asso. Prof. E. B. Univ. of Illinois, Urbana, Ill. 903 S. Busey Ave.

McAlister, Prof. E. H. Univ, of Oregon, Eugene, Ore, 334 Pearl St.

\$McAuley, P. H. Westinghouse Electric Co., E. Pittsburgh, Pa.

MCBERTy, Pres. F. R. North Electric Manufacturing Co., Galion, Ohio. 442 Lincoln $W a y, W$.

McCain, Prof. GerTrude I. State Coll., Redford, Va.

\$McCankIE, R. C. Equitable Life Insurance Co. of Iowa, Des Moines, Ia.

McCartuy, E. D. Instr., Pennsylvania State Coll., State College, Pa. Unversity Club.

McCarTY, A. L. Lowell High School, San Francisco, Callf.

† McCaulay, Dr. F. S. 7 Cranmer Road, Cambridge, England.

†McClenon, Prof. R. B. Grinnell Coll., Grinnell, la.

McConnel,, Dr. A. J. 38 Trinity Coll., Dublin, Ireland.

McCormick, Esther O. Barnard Coll., Columbia Univ, New York, N. Y.

McCormrck, Prof. J. T. Boston Coll., Chestnut Hill, Mass. 761 Harrison Ave., Boston, Mass. 
McCoy, Prof. Dorothy. Belhaven Coll., Jackson, Miss.

McCoy, N. H. Univ. of Iowa, Iowa City, Ia. 618 E. Burlington St.

McDaniel, Prof. W. R. Western Maryland Coll., Westminster, Md.

McDonald, Emma W. (Mrs. J. H.). 2401 Ellsworth St., Berkeley, Calif.

†MCDonnell, John. 84 Marlborough Ave., Ottawa, Canada.

McDonough, Laura F. Moravian Coll. for Women, Bethlehem, Pa.

McDougle, Edith A. Instr., Women's Coll., Univ, of Delaware, Newark, Del.

McEwen, Prof. G. F. Oceanographer, Scripps Inst., Univ. of California, La Jolla, Calif. Box 68

McEwen, Asst. Prof W. H. Mt. Allison Univ., Sackville, N. B., Canada.

McFartan, Asst. Prof. L. H. Univ. of Washington, Seattle, Wash. 5244 11th St.

McFarland, Asst. Prof. Elsie J. Univ. of Oklahoma, Norman, Okla. Faculty Exchange.

McGavock, MARTHA. Instr., Rockford Coll., Rockford, Ill.

McGaw, Prof. F. M. Cornell Coll., Mount Vernon, Ia.

McGiffert, Prof. Jimes. Rensselaer Polytechnic Inst., Troy, N. Y. 169 8th St.

McHugH, Dean P.J. Boston Coll., Chestnut Hill, Mass.

McKelvey, Asso. Prof. J. V. Iowa State Coll., Ames, la. 2117 Graeber St.

McKelvey, Martha M. (Mrs. J. V.). Instr., Iowa State Coll., Ames, Ia. 2117 Graeber St.

\$McLenEgan, D. W. Industrial Engineering Dept., General Electric Co., Schenectady, N.Y.

McMackin, Dr. F. J. 49 Vreeland Ave., Rutherford, N. J.

MCMASTER, A. S. Instr., Univ. of Colorado, Boulder, Colo.

McMillan, Prof. Mary B. 1341 S. Third St., Wisconsin Rapids, Wis.

MCNaIR, I. S. Univ. of Wisconsin, Madison, Wis.

MCNALLY, Rev. P. A. Director, Georgetown Coll. Observatory, Washington, D. C.

McShane, Dr. E. J. National Research Fellow. 1426 Touro St., New Orleans, La.

MacColl, L. A. Bell Telephone Laboratories, 463 West St., New York, N. Y.

MacCreadie, Asst. Prof. W. T. Bucknell Univ., Lewisburg, Pa. 1425 W. Market St.

MacDovgal, H. B. Instr., South Dakota State Coll., Brookings, S. Dak.

MACDuffeE, Asso. Prof. C. C. Ohio State Univ., Columbus, Ohio. 365 Oakland Park Ave.

Mackie, Asso. Prof. E. L. Univ. of North Carolina, Chapel Hill, N. C. 702 Ginghoul Road.

\$MacKrnnon, W. D. Equitable Life Insurance Co. of Iowa, Des Moines, Ia. MacMillan, Prof. W. D. Univ. of Chicago, Chicago, IIl.

MacNeish, Asso. Prof. H. F. Coll. of the City of New York, New York, N. Y. Scarsdale, N. Y.

MacQuenn, Asso. Prof. Marion L. Southwestern Coll., Memphis, Tenn.

Maddison, Dr. Isaber. The Croft, Martin's Dam, Wayne, Pa.

Maddox, Prof. A. C. State Normal Coll., Natchitoches, La, 180 Jefferson St.

Magnusson, Prof. C. E. Univ. of Washington, Seattle, Wash.

MaIer, Dr. William. International Research Fellow. Univ. of Chicago, Chicago, Ill.

MaIzlish, Prof. Israel. Centenary Coll., Shreveport, La.

\$Makarov, A. G. 114 Walnut Ave., Wayne, Pa.

Male, Asst. Prof. C. T. Union Coll., Schenectady, N. Y.

MalloRy, Asso. Prof. V. S. New Jersey State Teachers' Coll., Montclair, N. J. 351 Springdale Ave., East Orange, N. J.

Manchester, Prof. R. E. Kent State Coll., Kent, Ohio.

TManning, Asso. Prof. H. P. Brown Univ., Providence, R. I. 106 Carrington Ave.

MAnning, Prof. W. A. Stanford Univ., Stanford University, Calif. 649 Alvarado Row.

Manson, Prof. E. S. Ohio State Univ., Columbus, Ohio.

ManY, Asst. Prof. ANna E. Newcomb Coll., New Orleans, La.

March, Asso. Prof. H. W. Univ. of Wisconsin, Madison, Wis. 1825 Summıt Ave.

MARDEN, Dr. Morris. 566 Shirley St., Winthrop, Mass.

Maria, Asst. Prof. A. J. Univ. of Illinois, Urbana, Ill.

Marie Cecilia (MangoldD), Sister. Prof., Trinity Coll., Washington, D. C.

Marm, Prof. AnNa. Bethany Coll., Lindsborg, Kans.

Marriott, Prof. R. W. Swarthmore Coll., Swarthmore, Pa. 213 Lafayette Ave.

Marshall, Prof. William. Purdue Univ., Lafayette, Ind. 1017 State St.

MarTin, Prof. EmiLIE N. Mount Holyoke Coll., South Hadley, Mass. 
Martin, M. H. Instr., Johns Hopkins Univ., Baltimore, Md. 2821 St. Paul St.

Marvin, Prof. H. H. Univ. of Nebraska, Lincoln, Neb. Brace Laboratory.

Mary Bertrand (Walton), Sister. Marywood Coll., Scranton, Pa.

Mary Columba, Sister. Villa Maria Coll., Immaculat $1, \mathbf{P a}$.

Marslow, Al.eXander. Univ. of California, Berkeley, Calif. 2521 Piedmont Ave.

†MASON, Pres. Max. Rockefeller Foundation, 61 Broadway, New York, N. Y. Room 2701

Mason, Asso. Prof. T. E. Purdue Univ., Lafayette, Ind. 103 Waldron St.

Mason, Asst. Prof. W. E. Univ. of California at Los Angeles, Los Angeles, Calif.

Matheson, Prof. John. Queen's Univ., Kingston, Ontario, Canada.

Mathewson, Asst. Prof. L. C. Dartmouth Coll., Hanover, N. H.

May, Lida B. Box 783, Snyder, Texas.

\$MAYOR, J. R. Instr., Univ. of Wisconsin, Madison, Wis. North Hall.

MeACHAM, Prof. E. D. Univ. of Oklahoma, Norman, Okla. Faculty Exchange.

MEAD, F. B. Executive Vice-Pres., Lincoln National Life Insurance Co., Fort Wayne, Ind.

Mead, Mrs. Sallie P. Dept. of Development and Research, American Telephone and Telegraph Co., 195 Broadway, New York, N. Y.

Mears, Asst. Prof. Florence M. George Washington Univ., Washington, D. C. 512 Park Lane, Bethesda, Md.

Meder, Asst. Prof. A. E. New Jersey Coll. for Women, Rutgers Univ., New Brunswick, N. J.

Melcher, Dr. George. Asst Superintendent of Schools, Kansas City, Mo. Library Bldg.

Mendenhall, Pres. W. O. Friends Univ., Wichita, Kans.

Menge, W. O. Instr., Univ. of Michigan, Ann Arbor, Mich. 1307 Gardner Ave.

Mensenkamp, L. E. 521 W. Cottonwood St., Freeport, Ill.

MerrmL, Prof. A. S. Univ, of Montana, Missoula, Mont.

\$MerRILL, F. W. Engineering Dept., General Electric Co., Fort Wayne, Ind.

tMerril, Prof. Helen A. Wellesley Coll., Wellesley, Mass.

Merrinl, J. E. Instr., Princeton Univ., Princeton, N. J. 14 Prospect Ave.

Merriman, Asst. Prof. G. M. Univ. of Cincinnati, Cincinnati, Ohio.

Messrck, Prof. J. F. Emory Univ., Emory University, Ga.

†MetzleR, Dean W. H. New York State Coll. for Teachers, Albany, N. Y. 4 Glenwood St.

Meyer, Prof. H. A. Hanover Coll., Hanover, Ind.

MrCHAEL, Asso. Prof. A. D. California Inst. of Technology, Pasadena, Calif.

Michie, Prof. J. N. Texas Technological Coll., Lubbock, Tex. 2215 16th St.

Mrckelson, Prof. E. L. New Mexico State Teachers Coll., Silver City, N. Mex.

\$Middleton, C. W. Babcock and Wilcox Co., 85 Liberty St., New York, N. Y.

Midgley, Thomas. General Motors Chemical Co., Dayton, Ohio.

MILEs, Asso. Prof. E. J. Yale Univ., New Haven, Conn. 87 Marvel Road.

MIIES, Asst. Prof. E. R. C. Duke Üniv., Durham, N. C.

Miller, Dr. A. L. 25 Clinton Road, Brookline, Mass.

MIILER, Dr. Bessie I. Instr., Univ, of Illinois, Urbana, Ill. $1201 \mathrm{~W}$. Oregon St.

Miller, Prof. D. C. Case School of Applied Science, Cleveland, Ohio.

MILLER, E. A. 113 N. Tioga St., Ithaca, N. Y.

Mrller, E. W. Univ. of Michigan, Ann Arbor, Mich. 1930 Cambridge Road.

Miller, F. H. Instr., Columbia Univ., New York, N. Y. 579 Fort Washington Ave.

†Mmler, Prof. G. A. Univ. of Illinois, Urbana, Ill. 1203 W. Illinous St.

Mrller, Asst. Prof. H. L. Univ. of Cincinnati, Cincinnati, Ohio. Coll. of Engineering and Commerce.

Miller, Prof. I. L. South Dakota State Coll., Brookings, S. Dak. 718 12th Ave.

MILLER, Research Prof. J. A. Swarthmore Coll., Swarthmore, Pa.

MiLler, Prof. J. S. Emory and Henry Coll., Emory, Va.

Miller, Asso. Prof. Norman. Queens Univ., Kingston, Ontario, Canada.

Mrller, Asst. Prof. W. M. Marquette Univ., Milwaukee, Wis. 1597 Humboldt Blvd.

Mrls, Prof. C. N. State Normal Univ., Normal, Ill. 602 N. School St.

MiLNE, Dr. E. L. Lake Forest Academy, Lake Forest, Ill.

Mine, Asst. Prof. T. H. Univ. of Manitoba, Winn peg, Manitoba, Canada

MrNe, Prof. W. E. Univ. of Oregon, Eugene, Ore. Stanford Univ., Stanford University, Calhf.

MIINE, Prof. W. P. Leeds Univ., Leeds, England.

MIRICK, G, R. Lincoln School, 425 W, 123d St., New York, N. Y. 
Miser, Prof. W. L. Vanderbilt Univ., Nashville, Tenn. 1715 Ashwood Ave. MitchelL, Dr. A. K. Instr., Yale Univ., New Haven, Conn.

Mrtchell, Prof. H. H. Univ. of Pennsylvania, Philadelphia, Pa. College Hall. Mrtcheld, Prof. U. G. Univ. of Kansas, Lawrence, Kans. 1313 Massachusetts St. \$MrrchelL, W. C. Taft Fellow. 2390 Wheeler St., Univ. of Cincinnati, Cincinnati, Ohio.

Mode, Prof. E. B. Boston Univ., Boston, Mass. 688 Boylston St.

Morr, Pres. Henry. U. S. Life Insurance Co., 105 5th Ave., New York, N. Y.

Molina, E. C. Telephone Engineer, American Telephone and Telegraph Co., 195 Broadway, New York, N. Y.

Monskx, Morris. Mutual Life Insurance Co, 32 Nassau St., New York, N. Y. MontagUe, Harriet F. Instr., Univ. of Buffalo, Buffalo, N. Y.

Montgomery, P. V. Actuary, Southland Life Insurance Co., Dallas, Tex.

Moody, Dr. Ethel I. Sweet Briar Coll., Sweet Briar, Va.

Moody, Prof. W. A. Bowdoin Coll., Brunswick, Me. 60 Federal St.

MOORE, Prof. C. L. E. Massachusetts Inst. of Technology, Cambridge, Mass.

Moore, Prof. C. N. Univ. of Cincinnati, Cincinnati, Ohio 219 Woolper Ave.

†MoORE, Prof. E. H. Univ. of Chicago, Chicago, Ill. Hotel del Prado, 53d St. and Hyde Park Blvd.

Moore, Asst. Prof. L. T. Yale Univ., New Haven, Conn. 228 Park St.

MOORE, Prof. R. L. Univ, of Texas, Austin, Tex.

MOORE, Asst. Prof. T. W. Indiana Univ., Bloomington, Ind.

MoORE, Asst. Prof. W. L. Univ. of Louisville, Louisville, Ky. $4521 \mathrm{~S}$. Second St.

Moots, Prof. E. E. Cornell Coll., Mt. Vernon, Ia.

Morales, Prof. J. D. Univ. of Porto Rico, Mayaguez, Porto Rico.

MoRdell, Prof. L. J. Univ. of Manchester, Manchester, England.

Morehead, Prof. J. C. Carnegie Inst. of Technology, Pittsburgh, Pa.

Moreno, Prof. H. C. Stanford Univ., Stanford University, Calif., 684 Mirada Ave.

Morenus, Prof. Eugenie M. Sweet Briar Coll., Sweet Briar, Va.

Morgan, Dr. F. M. Director, Clark School, Hanover, N. H.

MoRI, Prof. TAtsuo. Naval Academy, Yedajima, Hiroshima-Ken, Japan.

MoRiarTy, M. M.S. Petersham, Mass.

Moritz, Prof. R. E. Univ. of Washington, Seattle, Wash. 4705 21st Ave., N. E.

Morley, Prof. Frank. Johns Hopkins Univ., Baltimore, Md. 2032 Park Ave.

MorLeY, Prof. R. K. Worcester Polytechnic Inst., Worcester, Mass.

MORRIL, Dr. W. K. Instr., Johns Hopkins Univ., Baltimore, Md. 5203 Gwynn Oak Ave.

Morris, Prof. C. C. Ohio State Univ., Columbus, Ohio.

MORRIS, EDWARD. Vice-President, Willits Water Co., Willits, Calif.

\$MORRIS, E. B. Travelers Insurance Co., Hartford, Conn.

Morris, Prof. F. R. State Coll., Fresno, Calif.

Morris, Asst. Prof. Max. Case School of Applied Science, Cleveland, Ohio.

Morris, Prof. Richard. Rutgers Univ., New Brunswick, N. J. 12 Johnson Si.

Morris, W. O. North American Life Insurance Co., 36 S. State St., Chicago, Ill.

Morrison, Montrord. Acme-International Co., $711 \mathrm{~W}$. Lake St., Chicago, Ill.

Morrow, Dr. D. C. Instr., Detroit City Coll., Detroit, Mich. 817 Mulney Ave., Winnpeg, Canada.

Morrow, E. B. Head Master, Gilman Country School, Roland Park, Baltimore, Md.

Morse, Asso. Prof. D. S. Union Coll., Schenectady, N. Y. 1372 Nott St.

Morse, Prof. Marston. Harvard Univ., Cambridge, Mass. 1737 Cambridge St.

Morton, Prof. A. B. Georgia School of Technology, Atlanta, Ga.

Moskovitz, David. Instr., Brown Univ., Providence, R. I.

Moss, J. H. Instr., New York Univ., New York, N. Y. 100 Washington Sq. E.

Mossman, Throza A. Instr., State Agric. Coll., Manhattan, Kans.

\$MotT-Smith, HAROLd. Research Laboratory, General Electric Co., Schenectady, N. Y.

Moulton, Prof. E. J. Northwestern Univ., Evanston, Ill. 1114 Colfax St.

Moulton, Prof. F. R. Director of Utilities Power and Light Corp., 327 S. La Salle St., Chicago, Ill.

\$Moultrop, I. E. Edison Electric Illuminating Co., 39 Boylston St., Boston, Mass.

$\$$ Moursund, A. F., JR. Brown Univ., Providence, R. I.

Mowbray, Prof. A. H. Univ, of California, Berkeley, Calif. 806 San Luis Road.

MoxLE, K. E. Somerville High School, Somerville, N. J. 457 Putnam Ave., Brooklyn, $N . Y$. 
Mueht man, Rev. Paul. Instr., St. Louis Univ., St. Louis, Mo.

MuIR, Sir Thomas. Elmcote, Rondebosch, South Africa.

Mullemeister, Dr. Hermance. Instr., Univ. of Washington, Seattle, Wash.

Muller, Dr. Jessie M. J. (Mrs.). University Station, Austin, Tex.

Mullings, M. E. Instr., Univ. of Cincinnati, Cincinnati, Ohio.

Mullins, Prof. G. W. Barnard Coll., Columbia Univ., New York, N. Y.

Murnaghan, Prof. F. D. Johns Hopkins Univ., Baltimore, Md. 6202 Sycamore Road.

MuRPhy, R. D. Equitable Life Assurance Society, 120 Broadway, New York, N. Y. 28 Godfrey Road, Upper Montclair, N.J.

Murray, Prof. D. A. McGill Univ., Montreal, Canada. 3653 University St.

Murray, Dr. F. H. American Telephone and Telegraph Co., 195 Broadway, New York, N. Y.

MurRay, Prof. V.F. Cataw'a Coll., Salisbury, N. C. Box 232.

Musselman, Prof. J. R. Coll. for Women, Western Reserve Univ., Cleveland, Ohio.

Nakss, Dr. Almar. Naval Academy of Norway, Horten, Norway.

Nassau, Asso. Prof. J. J. Case School of Applied Science, Cleveland, Ohio.

NeElley, Prof. J. H. Carnegie Inst. of Technology, Pittsburgh, Pa. 300 Broadmoor Ave., Sunset Hills, Mt. Lebanon.

\$NefF, Prof. I. F. Drake Univ, Des Moines, Ia. 2801 Brattleboro Ave.

NeIkrRk, Asst. Prof. L. I. Univ. of Washington, Seattle, Wash. 4723 21st Ave., N. E.

Nelson, Prof. A. L. Coll. of the City of Detroit, Detroit, Mich.

Nelson, Asso. Prof. C. A. Rutgers Univ., New Brunswick, N. J.

§Nelson, C. E. Missouri State Life Insurance Co., St. Louis, Mo.

Nelson, Asso. Prof. W. K. Univ. of Colorado, Boulder, Colo.

NewkiRK, Dr. B. L. Research Laboratory, General Electric Co., Schenectady, N. Y.

Newlin, Asst. Prof. R. L. Ohio Wesleyan Univ., Delaware, Ohio.

Newman, PhILIP. Instr., Townsend Harris Hall, Coll. of the City of New York, New York, N. Y. Furnald Hall, Columbia Unvv.

Newson, Prof. MarY W. (Mr. H. B.). Eureka Coll., Eureka, Ill.

Newron, Prof. G. A. Trinity Univ., Waxahachie, Tex.

Nichols, G. D. Instr., Univ. of Arkansas, Fayetteville, Ark.

Nichols, M. C. Engineer, Worcester Electric Light Co., Worcester, Mass. 37 Harvard St.

Nixon, ElizaberH E. (Mrs. J. C.). Instr., University School for Girls, Chicago, Ill. 5238 Woodlawn Ave.

\$Nixon, J. C. 2124 N. Hudson Ave., Chicago, Ill.

Noble, Prof. C. A. Univ. of California, Berkeley, Calif. 2224 Piedmont Ave.

NoEcker, J. L. Instr., Thiel Coll., Greenville, Pa.

\$Nollen, H.S. Equitable Life Insurance Co. of Iowa, Des Moines, Ia.

NoRLund, Prof. N. E. Univ. of Copenhagen, Copenhagen, Denmark. Malmogade 8.

NorthCotT, Asso. Prof. J. A. Columbia Univ., New York, N. Y.

Nowlan, Prof. F. S. Univ. of British Columbia, Vancouver, Canada.

Nowlan, Prof. Kevin. Spring Hill Coll., Spring Hill, Ala.

Nyswander, Asst. Prof. J. A. Univ. of Michigan, Ann Arbor, Mich. 3016 Angell Hall.

OAkley, Asst. Prof. C. O. Brown Univ., Providence, R. I.

O'Brien, Dean ALBERT. St. Bonaventure's Coll., St. Bonaventure, N. Y.

O'Brien, Prof. Katuerine E. Coll. of New Rochelle, New Rochelle, N. Y. Edgewood Hall, 246 Lockwood Ave.

O'Callaghan, Prof. Frank. Coll. of New Rochelle, New Rochelle, N. Y. 210 W. 107th St., New York, N. Y.

O'ConNell, J. E. 'St. John's Seminary, Little Rock, Ark, 2501 State St.

Ogburn, Prof. J. H. Lehigh Univ., Bethlehem, Pa. 625 Centre St

OGG, Dr. F. C. Univ. of Illinos, UUrbana, Ill. 905 1st St., Champaign, Ill.

Oglessy, Prof. E. J. Washington Square Coll., New York Univ., New York, N. Y. 4511 158th St., Flushing, $N$. $Y$.

Okada, Asst. Pr f. Yoshitomo. Tôhoku Imperial Univ., Sendai, Japan.

Ouds, Asst. Prof. E. G. Carnegie Inst. of Technology, Pittsburgh, Pa. 1424 Barnsdale St.

Olds, Pres. G. D. Amherst Coll., Amherst, Mass.

OtmSTEAd, Dr. P. S. Research Engineer, Bell Telephone Laboratories, 463 West St., New York, N. Y. 
OLson, Emma J. Instr., Northwestern Univ., Evanston, Ill. 724 Simpson St. OLson, Asst. Prof. H. L. Michigan State Coll., East Lansing, Mich. 544 Forest Ave. OpPEnherm, Alexander. 29 Rectory Road, Higher Crumpsall, Manchester, England.

Ore, Prof. Oystein. Yale Univ., New Haven, Conn. Harkness Hall 323.

Osbors, Dr. J. O. Harris Teachers Coll, St. Louis, Mo. 3239 Lafayette Ave. OSGOoD, Prof. W.F. Harvard Univ., Cambridge, Mass. 74 Avon Hall St.

O'Shaughnessy, Prof. Lours. Virginia Polytechnic Inst., Blacksburg, Va. Box 177.

§OTıs, W. H. Pacific Mutual Life Insurance Co., Los Angeles, Calif.

OTT, Prof. W. P. Univ. of Alabama, University, Ala.

OTteN, HenRY. Interborough Rapid Transit Co., 600 W. 59th St., New York, N. Y. Overman, Prof. J. R. State Normal Coll., Bowling Green, Ohio.

Owens, Prof. F. W. Pennsylvania State Coll., State College, Pa. 526 E. Foster Ave. Owens, Dr. Helen B. (Mrs. F. W.). 526 E. Foster Ave., State College, Pa. Owens, H. D. Instr., Univ, of Minnesota, Minneapolis, Minn.

PaAswell, George. Civil Engineer. 1950 Andrews Ave., Bronx, New York, N. Y. PAGE, Prof. J. M. Univ. of Virginia, University, Va.

Page, Prof. Leigh. Yale Univ., New Haven, Conn. Sloane Physics Laboratory.

§aine, W. S. Aetna Life Insurance Co., Hartford, Conn.

PALL, Dr. GoRdon. National Research Fellow. California Inst. of Technology, Pasadena, Calif.

PaLMER, Dean C. I. Armour Inst. of Technology, Chicago, IIl.

Paradiso, L. J. Instr., Cornell Univ., Ithaca, N. Y.

tPARANJPYe, Dr. R. P. India Office, London S. W. 1, England.

\$PARK, R. H. Engineering General Dept., General Electric Co., Schenectady, N. Y. PARK, Prof. R. S. Eastern Teachers Coll., Richmond, Ky. Burnam Court.

\$PARKER, Asst. Prof. W. V. Univ. of North Carolina, Chapel Hill, N. C.

Parkinson, Asso. Prof. G. A. Univ. of Wisconsin, Extension Division. 619 State St., Milwaukee, Wrs.

Parsons, C. H. Townsend, Va.

†PArTridge, Dr. E. A. West Philadelphia High School, 48th and Walnut Sts., Philadelphia, $\mathrm{Pa}$.

Patten, W. E. 401 Conklin Ave., Binghamton, N. Y.

Patterson, Asso. Prof. B. C. Hamilton Coll., Clinton, N. Y. College Hull. Pattillo, Prof. N. A. Randolph-Macon Woman's Coll., Lynchburg, Va.

PAUL, W. R. Royal Society of Arts, 327 W. 69th St., Chicago, III.

Paxton, Asso. Prof. E. K. Washington and Lee Univ., Lexington, Va.

PAyne, C. K. Instr., New York Univ., New York, N. Y. 100 Washington Sq. E. Pegram, Prof. AnNre M. Greensboro Coll., Greensboro, N. C.

Pehrson, Asso. Prof. E. W. Univ, of Utah, Salt Lake City, Utah.

Pell-Whegler, Anna. See Wheeler, Anna Pell.

Pellétrer, Prof. Arthur. Polytechnic School of Montreal, Montreal, Canada. 8456 Drolet St.

PEND ETon, Pres. Elden F. Wellesley Coll., Wellesley, Mass.

Pennell, W. O. Southwestern Bell Telephone Co., St. Louis, Mo.

PEPPER, Dr. Echo D. Univ. of Illinois, Urbana, Ill.

Perkins, Dr. F. W. Instr., Dartmouth Coll., Hanover, N. H.

Perkins, H. A. Hampton Inst., Hampton Institute, Va.

PErrin, O. W. Penn Mutual Life Insurance Co., 6th and Walnut Sts., Philadelphia, $\mathrm{Pa}$.

§Peters, J. F. Consulting Engineer, Westinghouse Electric Co., E. Pittsburgh, Pa.

Perers, Dr. J. W. Instr., Univ. of Illinois, Urbana, Ill. 360 Math. Bldg.

Peterson, J. K. 1915 Blakemore Ave., Nashville, Tenn.

Peterson, Prof. O. J. State Teachers Coll., Emporia, Kans.

Peterson, T. S. Univ, of Michıgan, Ann Arbor, Mich.

§Petrovsky, Vyacheslav. Theoretical Research Engineer, General Electric Co., West Lynn, Mass. 23 Commercial St.

Petrit, Prof. H. P. Marquette Univ., Milwaukee, Wis. 1664 Bartlett Ave.

Preiffer, Asst. Prof. G. A. Columbia Univ., New York, N. Y. Palisade, N. J. Phalen, Prof. H. R. St. Stephens Coll., Annar dale-on-Hudson, N. Y.

Phillips, Asst. Prof. H. B. Massachusetts Inst. of Technology, Cambridge, Mass.

PhIpps, Asso. Prof. C. G. Univ, of Florıda, Gainesville, Fla. Box 2132, University Station. 
Pierce, Prof. T. A. Univ. of Nebraska, Lincoln, Neb. 1811 Pepper Ave.

Pierpont, Prof. James. Yale Univ., New Haven, Conn. 102 A von St.

PIXLEY, H. H. Instr., Coll. of the City of Detroit, Detroit, Mich.

Plant, Prof. L. C. Michigan State Coll., East Lansing, Mich.

Plimpton, Dr. G. A. Ginn and Co., 70 5th Ave., New York, N. Y.

Pogo, Dr. Alexander. 189 Harvard Library, Cambridge, Mass.

Pokorny, Oldrich. Univ. of Prague, Prague, Czechoslovakia.

Poltard, Asst. Prof. H. S. Miami Univ., Oxford, Ohio.

Pollock, Asst. Prof. Saul. Univ. of California, Berkeley, Calif.

Pooler, L. G. Instr., Hunter Coll., Park Ave. and 68th St., New York, N. Y. 456 Riverside Drive.

Poor, Prof. C. L. Columbia Univ., New York, N. Y. 35 E 69th St

Poor, Prof. J. M. Dartmouth Coll., Hanover, N. H. Shattuck Observatory.

Poor, Asso. Prof. V. C. Univ of Michigan, Ann Arbor, Mich. 930 Packard St.

PorITsky, Dr. Hillel. General Electric Co, Schenectady, N. Y. 1011 Union St.

PorTer, Asso. Prof. C. S. Amherst Coll., Amherst, Mass Box 733, 9 Hutchcock Road.

PORTER, E. A. Actuary 3d Floor, 910 Bergen Ave., Jersey City, N. J.

PorTer, Prof. M. B. Univ, of Texas, Austin, Tex. 310 E. 21st St.

PorTer, Prof W. L. Agric. and Mech. Coll, College Station, Tex.

PosEY, F, D. 570 Florida, Buenos Aires, Argentina.

Post, Dr. E L. 135 Terrace View Ave., New York, N. Y.

Pound, Prof. V. E. Univ. of Buffalo, Buffalo, N. Y. Hayes Hall.

Pounder, Asst. Prof. I. R. Univ. of Toronto, Toronto, Canada.

Poweld, J. E. Instr., Michigan State Coll, East Lansing, Mich

†Powers, R. E. Denver and Rio Grande Railroad, 214 Equitable Bldg., Denver, Colo.

Prasad, Prof. Ganesh. Calcutta Univ., Calcutta, India. Corporation St., 2 Samanaya Mansions.

\$Pratt, A. D. Babcock and Wilcox Co., 85 Liberty St., New York, N. Y.

\$PRATT, Pres. A. G. Babcock and Wilcox Co., 85 Liberty St., New York, N. Y.

Preston, Bernard. Certified Public Accountant. 50 E. 42d St., New York, N. Y.

Pretz, Prof. P. S. St. Benedict's Coll., Atchison, Kans.

PrICE, G. B. Instr., Harvard Univ., Cambridge, Mass. 34 Mellen St.

Price, IR ENE. Instr., State Teachers Coll., Oshkosh, Wis.

\$PrINCE, D. C. Research Engineer, Research Laboratory, General Electric Co., Schenectady, N. Y.

Pummill, Prof. L. E. State Coll., Springfield, Mo.

†PuPIN, Prof. M. I. Columbia Univ., New York, N. Y.

Putnam, Asso. Prof. R. G. New York Univ., New York, N. Y. Bridge and Church Sts., Tarrytown, N. Y.

Putnam, Dean T. M. Univ. of California, Berkeley, Calif.

Pule, Prof. H. R. Penn College, Oskaloosa, Ia.

Quigley, Mary J. Boston Teachers Coll., Boston, Mass. 72 Grampian Way, Dorchester, Mass.

RAFFERTY, T. C. International Life Insurance Co., 1325 International Life Bldg., St. Louis, Mo

Ragsdale, Prof. Virginia. Coll. for Women, Greensboro, N. C. Jamestown, $N . C$.

RAIFord, Dr. T. E. Univ. of Michigan, Ann Arbor, Mich. 1618 Shadford Road.

Rainich, Asso. Prof. G. Y. Univ, of Michigan, Ann Arbor, Mich

RaINville, E. D. Instr., Univ. of Colorado, Boulder, Colo. 1005 12th St.

Rambo, Asso. Prof. Susan M. Smith Coll., Northampton, Mass. 12 Barrett Place.

Ramler, Prof. O. J. Catholic Univ., Washington, D. C. 12 Girard St., N. E.

RamsDell, Prof G. E. Bates Coll., Lewiston, Me. $40 \mathrm{Mt}$. Ave.

RankIN, Prof. J. M. Coll. of Idaho, Caldwell, Id. Box 97, R. F. D. 4.

Rankin, Prof. W. W. Duke Univ., Durham, N. C.

Ransom, Prof. W. R. Tufts Coll, Tufts College, Mass, 29 Sawyer Ave.

Ranum, Prof. Arthur. Cornell Univ., Ithaca, N. Y. 3 Central Ave.

§RAShevsky, Nicholas. Research Engineering Dept., Westinghouse Electric Co., E. Pittsburgh, Pa.

RAsmuSen, Ruth B. Instr., South Dakota State Coll, Brookings, S. Dak.

Rasor, Prof. S. E. Ohio State Unıv, Columbus, Ohio. 1594 Nenll Ave.

RAU, Dean A. G. Moravian Coll., Bethlehem, Pa. 38 W. Market St.

Raudenbush, H, W. Instr., Columbıa Univ., New York, N. Y. Hamalton Hall. 
Rawles, Dr. T. H. Instr., Yale Univ., New Haven, Conn. 399 Yale Station. RAwlins, Asso. Prof. C. H. 'U. S. Naval Ácademy, Annapolis, Md. 13 Franklin St. Raynor, Asso. Prof. G. E. Univ. of Oklahoma, Norman, Okla. Faculty Exchange. Reaves, Dean S. W. Univ, of Oklahoma, Norman, Okla.

REChard, Asso. Prof. O. H. Univ, of Wyoming, Laramie, Wyo. $269 \mathrm{~N} .8 \mathrm{th}$ St. $\nmid$ REDDICK, Prof. H. W. Cooper Union, New York, N. Y.

Reed, Asso. Prof. F. W. Ohio Univ., Athens, Ohio.

ReED, Prof. L. J. Johns Hopkins Univ., Baltimore, Md. $615 \mathrm{~N}$. Wolfe St.

Reen, Asst. Prof. C. G. Gettysburg Coll., Gettysburg, Pa. 25 Chambersburg St. ReEs, Asso. Prof. C. J. Univ. of Delaware, Newark, Del.

ReEs, Mina S. Instr., Hunter Coll., Park Ave. and 68th St., New York, N. Y. REEs, W. A. Instr., Houston Junior Coll, Houston, Tex.

ReEve, Prof. W. D. Teachers Coll., Columbia Univ., New York, N. Y. $525 W$. 120 th St.

Reid, Prof. L. W. Haverford Coll., Haverford, Pa.

REID, Dr. W. T. National Research Fellow. Úniv, of Chicago, Chicago, Ill. 6017 Kimbark Ave.

Renlu, Prof. J. F. Univ. of Iowa, Iowa City, Ia. 212 Physics Bldg.

Reinsch, Prof. B. P. Southern Methodist Univ., Dallas, Tex. School of Engineering.

Remick, Prof. B. L. State Agric. Coll., Manhattan, Kans. 613 Houston St.

Remington, P. E. Mountain States Telephone and Telegraph Co., Denver, Colo. Box 960 .

Reynolds, Asso. Prof. C. N. Univ. of West Virginia, Morgantown, W. Va. 217 McLane Ave.

Reynolds, Prof. F. G. Coll. of the City of New York, New York, N. Y.

REYNolds, Prof. J. B. Lehigh Univ., Bethlehem, Pa. 721 Broad St.

RHodes, C. E. Instr, Univ. of Cincinnati, Cincinnati, Ohio.

Rhodes, E. E. Mutual Benefit Life Insurance Co., 750 Broad St., Newark, N. J.

Riberio, Prof. L. J. F. 456 Rua Augusta, Pernambuco, Brazil.

RICE, C. M. 19 Pearl St., Worcester, Mass.

\$RICE, C. W. Research Engineer. 3 Lowell Road, Schenectady, N. Y.

RICE, Prof. HARRIS. Worcester Polytechnic Inst., Worcester, Mass. Station C.

RICE, Asso. Prof. J. N. Catholic Univ., Washington, D. C. 3326 13th St., N. E.

RICE, Asst. Prof. L. H. Massachusetts Inst. of Technology, Cambridge, Mass. Room 2-177.

Richardoson, Prof. A. R. University Coll., Singleton Park, Swansea, Wales.

Richardson, Prof. A. V. Bishop's Coll., Lennoxville, Quebec, Canada.

Richardson, D. P. Instr., Univ. of Arkansas, Fayetteville, Ark.

Richardson, Asst. Prof. M. R. Georgia School of Technology, Atlanta, Ga. 763 Greenwood Ave.

$\nmid$ Richardson, Dean R. G. D. Brown Univ., Providence, R. I.

RicherT, Prof. D. H. Bethel Coll., Newton, Kans.

Richeson, Asst. Prof. Altie W. Univ, of Maryland, Baltimore, Md. 306 E. $32 d$ St.

RICHmond, Asst. Prof. D. E. Williams Coll., Williamstown, Mass. Box 696.

$\nmid$ Rrchmond, Dr. H. W. King's Coll., Cambridge, England.

RICkER, Dr. N. H. Consulting Physicist. Box 1619, Houston, Tex.

Rrder, Asso, Prof. P. R. Washington Univ., St. Louis, Mo. Yale Univ., Nere Haven, Conn.

RIETz, Prof. H. L. Univ. of Iowa, Iowa City, Ia. Physics Bldg.

RIETz, J. C. Midland Mutual Life Insurance Co., Columbus, Ohio. Box 1402.

RIGgS, Prof. N. C. Carnegie Inst. of Technology, Pittsburgh, Pa.

Rines, David. Patent Attorney. 20 Kilby St., Boston, Mass.

Risley, Prof. W. J. Colorado School of Mines, Golden, Colo. 1522 Maple St.

Risselman, Prof. W. C. Arizona State Teachers Coll., Flagstaff, Ariz.

Ritr, Asso. Prof. J. F. Columbia Univ., New York, N. Y. 300 W. 108th St.

Robbins, Dr. R. B. Vice-Pres., Union Labor Life Insurance Co., Washington, D. C. Machinists Bldg.

Robert, Prof. H. M. U. S. Naval Academy, Annapolis, Md. 53 Southgate Ave. RoberTs, Prof. B. D. Parsons Coll., Fairfield, Ia. 409 W. Carpenter St.

RoBerts, Adj. Prof. J. H. Univ. of Texas, Austin, Tex. University Station. RoberTs, Dean Maria M. Iowa State Coll., Ames, Ia. Room 104, Central Bldg.

Robertson, Asst. Prof. H. P. Princeton Univ., Princeton, N. J. D-1 Prospect A pts.

§Robinson, E. L. Turbine Engineering Dept., General Electric Co., Schenectady, N. Y. 
Robinson, Prof Georgia E. Junior Coll., Centerville, Ia

Robinson, Prof. H. A. Agnes Scott Coll., Decatur, Ga.

Robinson, L. B. 131 E. North Ave., Baltimore, Md.

Robinson, Adj. Prof. L. V. Texas Technological Coll., Lubbock, Tex.

Robinson, Asso. Prof. P. G. Iowa State Coll., Ames, Ia.

RoBinson, Dr. Robin. Instr., Dartmouth Coll., Hanover, N. H. 21 Prospect St.

RoBison, Asst. Prof. G. M. Duke Univ., Durham, N. C. Trinity Coll.

Rodgers, Prof. T. G. New Mexico Normal Univ., Las Vegas, N. Mex. 1018 4th St., Easi Las Vegas.

$\nmid$ Roe, Dr. JosepHINe R. (Mrs. E. D., JR.). 123 W. Ostrander Ave., Syracuse, N. Y. ROESER, H. M. Bureau of Standards, Washington, D. C.

RoessLer, Dr. E. B. Instr., Univ. of California, Berkeley, Calif. 420 Wheeler Hall. Roever, Prof. W. H. Washington Univ., St. Louis, Mo.

Roman, Dr. InwIN. Mathematical Physicist, 90 Valley Way, West Orange, N. J.

RomIG, H. G. Statistician, Bell Telephone Laboratories, 463 West St., New York, N. Y. Engineervng Dept.

Roonex, Prof. J. A. Mount St. Charles Coll., Helena, Mont.

Roos, Asst Prof. C. F. Cornell Univ., Ithaca, N. Y. 200 College Ave.

Roosevelt, G. E. 30 Pine St., New York, N. Y.

Roor, Prof. R. E. U. S. Naval Academy, Annapolis, Md. 7 Franklin St.

Rorer, Dr. J. T. 333 N. 34th St., Philadelphia, Pa.

Rose, C. E. Electrical Engineer, Arkansas Cold Storage Co., Little Rock, Ark.

Roserrugh, Prof. T. R. Univ. of Toronto, Toronto, Canada. 92 Walmer Road.

Rosenbach, Asso. Prof. J. B. Carnegie Inst. of Technology, Pittsburgh, Pa. 5836 Alderson St.

Rosenbaum, Dr. Joseph. Instr., Milford School, Milford, Conn.

Rosenbaum, S. B. Director, Milford School, Milford, Conn. 56 Gulf St.

Rosinger, Dr. K. E. Harvard Univ., Cambridge, Mass.

Ross, ARnold E. Univ. of Chicago, Chicago, Ill. 5525 Drexel Ave.

Ross, C. M. Cheltenham Coll., Cheltenham, England.

RorH, Asst. Prof. W. E. Univ. of Wisconsin at Milwaukee, Milwaukee, Wis. 619 State St.

Rothrock, Dean D. A. Indiana Univ., Bloomington, Ind. 1000 Atwater Ave.

Roulton, J. A. Mechanical Engineer. 1331 Washington St., Wilmington, Del.

Rouse, Asst. Prof. L. J. Univ. of Michigan, Ann Arbor, Mich. 1137 Michigan Ave.

Rowe, Prof. C. H. Univ. of Dublin, Dublin, Ireland. 38, Trinity Coll.

RowE, Pres. J. E. Clarkson Memorial Inst. of Technology, Potsdam, N. Y.

Rowland, Prof. S. A. Ohio Wesleyan Univ., Delaware, Ohio. 49 Oak Hill Ave.

Royce, G. L. Instr., Racine Military Acad., Racine, Wis.

Ruger, Asso. Prof. H. A. Educational Psychologist. Teachers Coll., Columbia Univ., New York, N. Y. 41 Claremont Ave.

Runge, Asst. Prof. LuLu L. Univ. of Nebraska, Lincoln, Neb.

Running, Prof. T. R. Univ. of Michigan, Ann Arbor, Mich. 1019 Michigan Ave.

RUPP, Asst. Prof. C. A. Pennsylvania State Coll., State College, Pa.

Rusk, Prof. W. J. Grinnell Coll., Grinnell, Ia. 1415 Park St.

Russeld, Prof. W. P. Pomona Coll., Claremont, Calif.

RutledGe, Asso. Prof. GeORGE. Massachusetts Inst. of Technology, Cambridge, Mass. 29 Bellevue Road, Belmont, Mass.

Rutr, Dr. N. E. National Research Fellow. Univ. of Texas, Austin, Tex. $408 \mathrm{~W}$. 33d St.

§Ryan, R. A. Treasurer, Western and Southern Life Insurance Co., 4th and Broadway, Cincinnati, Ohio.

\$SACHER, AnNa. 1840 Lacombe Ave., New York, N. Y.

†SAFFord, Prof. F. H. Univ. of Pennsylvania, Philadelphia, Pa. College Hall.

SaIBEL, Asst. Prof. E. A. Carnegie Inst. of Technology, Pittsburgh, Pa.

Sr. JoHn, Dr. C. E. Mt. Wilson Observatory, Pasadena, Calif.

Sakellariou, Prof. Nilos. Univ. of Athens, Athens, Greece. Rue Voulgaroctonou $22 a$.

Salas-Edwards, Prof. Ramon. Univ. of Chile, Santiago, Chile.

Sanchez-Draz, Rafael. Instr., Univ. of Porto Rico, Mayaguez, Porto Rico, Coll. of Agric. and Mech. Arts, Box 231.

SANGER, R. G. Instr., Univ. of Chicago, Chicago, Ill. 116 S. Grove Ave., Oak Park, Ill

Sarafoglou, A. G. Robert Coll., Bebec, Stamboul, Turkey. 
Saunders, Farth. Maysville, DeKalb County, Mo.

Saurel, Prof. P. L. Coll. of the City of New York, New York, N. Y.

§Sauté, Asst. Prof. George. Cleveland Coll., Cleveland, Ohıo.

Scarborough, Asso Prof. J. B. U. S. Naval Academy, Annapolis, Md. Box 332.

SCarborough, Prof. J. H. State Teachers Coll., Warrensburg, Mo.

SchelkunofF, Dr. S. A. Bell Telephone Laboratories, 463 West St., New York, N. Y.

Schlauch, Helen M. Hunter Coll., Park Ave. and 68th St, New York, N. Y. 219 Diviszon Ave., Hasbrouck Heights, N. J.

SchlaUCH, Asst. Prof. W. S. New York Univ., New York, N. Y.

†Schmieder, Oscar. San Blas Ave., R. F. D No. 1, Atascadero, Calif.

SchnurmanN, Dr. Paul. 5914 Margaretta St., Pittsburgh, Pa.

Schoonmaker, Asst. Prof. Hazel E. New Jersey Coll. for Women, New Brunswick, N. J.

Schroeder, Prof. C. E. Boston Coll., Chestnut Hill, Newton, Mass.

SchuG, H. L. Research Engineer, Hoover Co., N. Canton, Ohio.

Schultz, Prof. Henry. Univ. of Chicago, Chicago, Ill. Socral Science Research Bldg.

SchuYler, Elmer. Bay Ridge High School, 67th St. and 4th Ave., Brooklyn, N. Y.

Schwartz, Dr. P. D. Care of Henry L. Doherty and Co., 60 Wall St., New York, N. Y.

Schwat, Emeritus Prof. I. J. Univ. of Pennsylvania, Philadelphia, Pa. 1226 S. 58th St.

Sch WEITZER, Dr. A. R. 452 Oakdale Ave., Chicago, Ill.

Schwertzer, E. O. Commonwealth Edison Co., Chicago, Ill 730 Waveland Ave.

Scotr, Prof. Charlotte A. Bryn Mawr Coll., Bryn Mawr, Pa. 2 Storey's Way, Cambrudge, England.

SeArcy, Asst. Prof. C. L. Univ. of Nevada, Reno, Nevada.

\$SEDGwICK, C. H. W. Instr., Brown Univ., Providence, R. I.

SEE, Prof, T. J. J. Government Astronomer. Mare Island, Calif.

SeEly, Dr. Caroline E. 501 W. 116th St., New York, N. Y.

DE St́guier, Rev. J. 114 rue du Bac, Paris, 7e, France.

SEIDLIN, Prof. Joseph. Alfred Univ., Alfred, N. Y.

Seiverling, Prof. I. F. State Teachers College, Millersville, Pa.

SELLERs, EDWARD Actuary, Woodard, Fondillar and Ryan, 75 Fulton St., New York, N Y.

Sellew, Prof G. T. K̇nox Coll., Galesburg, Ill.

Serghiesco, Dr. Stefan. Columbia Univ., New York, N. Y. 301 Hamilton Hall. Seweld, Pres J.S. Alabama Marble Co., Birmingham, Ala 2801 Mountain Ave Shane, Dr. C. D. Instr., Univ. of California, Berkeley, Calif. 1111 Fresno Ave.

\$SHaPley, Prof HaRLow Harvard Univ, Cambridge, Mass. Harvard Observatory. SharP, J. G. Woodward, Fondiller and Ryan, 75 Fulton St., New York, N. Y.

Sharpe, Prof. F R. Cornell Univ., Ithaca, N Y 29 White Hall.

Shaud, Asst. Prof. H. C. Washington-Jefferson Coll., Washington, Pa.

SHAw, Asst. Prof. A. A. Univ of Arizona, Tucson, Ariz.

Shaw, Ass s. Prof. A. N. McGill Univ., Montreal, Canada. Physics Bldg.

SHaw, Prof. J. B. Univ. of Illinois, Urbana, Ill. Box 644, Station A, Champaign, Ill.

Shaw, Vice-Pres. W. F. Consulting Engineer, American Rio Grande Land and Irrigation Co., Mercedes, Tex.

Sheffer, Asso. Prof. H. M. Harvard Univ., Cambridge, Mass. Emerson Hall.

Shefrer, Asst. Prof. I. M. Pennsylvania State Coll., State College, Pa. $131 \mathrm{~W}$. Park Ave

Sheldon, Prof. E. W. Univ. of Alberta, Edmonton, Canada.

Shenton, Prof. W F. American Univ,, Washington, D. C. 3605 Porter St., N.W.

Sherer, Prof. C. R. Texas Christian Úniv., Fort Worth, Tex.

Sherwood, Prof. G. E. F. Univ. of California at Los Angeles, Los Angeles, Calif. 920 Hyperion Ave.

Shewhart, Dr. W. A. Engineer, Bell Telephone Laboratories, 463 West St., New York, N. Y.

SHirk, Prof. J. A. G. State Teachers Coll., Pittsburg, Kans.

Shively, Asso. Prof. L. S. Ball State Teachers Coll., Muncie, Ind. 407 Riverside Ave.

Shoemaker, Prof. H. M. North East High School, Philadelphia, Pa.

Shohat, Dr. J. A. Lecturer, Univ. of Pennsylvania, Philadelphia, Pa.

Shook, Asst. Prof C. A. Yale Univ., New Haven, Conn. 108 Furst Ave., West Haven, Conn. 
Sноoк, R. C. 5485 Ellis Ave., Chicago, Ill.

Short, R. L. Ashland School, 3921 N. Newstead Ave., St. Louis, Mo.

Shover, C. Grace. Instr., Connecticut Coll. for Women, New London, Conn.

Showman, H. M. Recorder and Lecturer, Univ, of California at Los Angeles, Los Angeles, Calif. 912 Hyperion Ave

Shriver, L. D. Charleroi High School, Charleroi, Pa. Bentleyville, Pa.

ShugerT, Prof. S. P. Univ. of Pennsylvania, Philadelphia, Pa. College Hall.

Shull, Prof. C. E. Bridgewater Coll., Bridgewater, Va.

Shuman, J. W. Consulting Engineer, Power Engineering Co., Minneapolis, Minn. 716 Metro. Life Bldg.

Shumway, Asso. Prof. R. R. Univ. of Minnesota, Minneapolis, Minn. 3844 Thomas Ave., $S$.

Shuster, Asst. Prof. C. N. State Teachers Coll , Trenton, N. J.

Sibert, Asst. Prof. H. W. Univ. of Cincinnati, Cincinnati, Ohio. Coll of Engrneering and Commerce.

Siceloff, Asso. Prof. L. P. Columbia Univ., New York, N Y.

Silberfarb, Asst. Prof. Samuel. Univ. of Akron, Akron, Ohio.

\$SIL BERSTEIN, Dr. LuDwig Eastman Kodak Co., Rochester, N. Y. 129 Seneca Parkway

Silverman, Asst. Prof. L. L. Dartmouth Coll., Hanover, N. H.

Simmons, Asso. Prof. H. A. Northwestern Univ., Evanston, Ill.

Simon, L. G. Statistician. 225 W. 34th St., New York, N. Y.

Simon, Prof. W. G. Western Reserve Univ., Cleveland, Ohio.

Simons, Prof. Lao G. Hunter Coll., Park Ave. and 68th St., New York, N. Y. 180 W. 88 th St.

Simpson, Prof. T. M. Univ. of Florıda, Gainesville, Fla. $547 \mathrm{~S}$. 9 th $\mathrm{St}$.

Simpson, Prof T McN. Randolph-Macon Coll., Ashland, Va.

Sinclarr, Prof Mary E. Oberlin Coll, Oberlin, Ohio. 260 Oak St.

Singer, JAMES. Instr., Princeton Univ., Princeton, N. J. 172 Nassau St.

Sinnott, R. V. 30 Harbison Ave., Hartford, Conn.

SiroKy, Asso. Prof. EDMOND. Washington Univ., St. Louis, Mo.

†Sisam, Prof. C. H Colorado Coll, Colorado Springs, Colo. $816 \mathrm{~N}$. Weber St.

\$Skeats, W. F Electrical Engineer, General Electric Co, Schenectady, N. Y. Bldg. $258 \mathrm{~A}$.

SkILEs, Prof. W. V. Georgia School of Technology, Atlanta, Ga. 103 Springdale Road.

Skinner, Prof E. B. Univ of Wisconsin, Madison, Wis. 210 Lathrop St.

Skolnik, David. Instr., Central High School, Newark, N. J. 67 Sunset Ave., Glen Rudge, N.J.

SLADE, J. J. Instr., Univ. of North Carolina, Chapel Hill, N. C.

SlAUGHT, Prof. H. E. Univ. of Chicago, Chicago, Ill. 5548 Kenwood Ave.

Slepian, Dr. JosePH Electrical Engineer, Westinghouse Electric Co., E Pittsburgh, $\mathrm{Pa}$.

†Slichter, Dean C. S. Univ, of Wisconsin, Madison, Wis. $636 \mathrm{~N}$. Frances St. Slobin, Prof. H. L. Univ. of New Hampshire, Durham, N. H.

SlotNICK, Dr M. M. 7 Otisfield St., Roxbury, Mass.

Small, Prof. L. L. Lehigh Univ., Bethlehem, Pa.

SmIley, Asst. Prof. C. H. Brown Univ, Providence, R. I.

Smith, A. H. Instr., Swarthmore Coll , Swarthmore, Pa.

SмIтн, Prof. A. W. Colgate Univ., Hamilton, N. Y.

Smrrt, Prof. C. D. Louisiana Coll., Pineville, La.

SмTTH, C. E. Chabot Observatory, 4917 Mountain Blvd., Oakland, Calif.

†Smith, Prof. Clara E. Wellesley Coll., Wellesley, Mass. 14 Waban St.

Smitr, Dean C. W. State Teachers Coll., Superior, Wis.

†Smith, Prof. D. E. Teachers Coll., Columbia Univ., New York, N. Y. 501 W. 120 th St.

Smith, Asso. Prof. D. M. Georgia School of Technology, Atlanta, Ga.

Smith, Prof. E. R. Iowa State Coll., Ames, Ia. Box 332, Station A.

Smith, Asst. Prof. Gertrude. Vassar Coll., Poughkeepsie, N. Y.

SmItH, Asso. Prof. G. W. Univ. of Kansas, Lawrence, Kans. 1730 Illinors St.

Smith, Helen F. Instr., Iowa State Coll., Ames, Ia. 113 Welch Ave.

Smith, Hartley L. Research Engineer, New England Power Construction Co., 89 Broad St., Boston, Mass.

Smith, Asso. Prof. Herman L. Louisiana State Univ., Baton Rouge, La. Universtty Station, Box 523. 
Smith, Asst. Prof. I. W. North Dakota Agric. Coll., Fargo, N. Dak. 112613 th St., $N$. \$SмIтH, J. C. Induction Motor Dept., General Electric Co., Schenectady, N. Y. §SмIтH, Dr. J. J. Electrical Engineer, General Engineering Laboratory, General Electric Co., Schenectady, N. Y.'

Smith, Mary F. College Recorder, Wellesley Coll., Wellesley, Mass.

Smith, Asst. Prof. P. A. Barnard Coll., Columbia Univ., New York, N. Y.

Smith, Prof. P. F. Yale Univ., New Haven, Conn. Box 10.

SmITH, Asst. Prof. R. F. Coll. of the City of New York, New York, N. Y. $463 \mathrm{~W}$. 144th St.

Smith, Asso. Prof. R. G. Kansas State Teachers Coll., Pittsburg, Kans.

SmITn, Prof. T. F. Little Rock Coll., Pulaski Heights, Little Rock, Ark.

Smith, Asst. Prof. T. L. Carnegie Inst. of Technology, Pittsburgh, Pa.

Smith, Dr. W. B. 9 Price Ave., Columbia, Mo.

SmITH, Prof. W. M. Lafayette Coll., Easton, Pa.

Smouroff, Prof. Alexander. Electrotechnical Inst., Leningrad 22, Russia. Pessanotchnaja 5 , log. 8.

Snedecor, Asso. Prof. G. W. Iowa State Coll., Ames, Ia. 807 Hodge Ave.

SNoor, T. E. Architect and Engineer. 50 Vesey St., New York, N. Y.

SNYDER, Asst. Prof. A. D. Union Coll., Schenectady, N. Y. 1592 Union St.

SNyder, Prof. M. B. Central High School, Phladelphia, Pa. 120 Woodside Ave., Narberth, $P a$.

SNyder, Prof. VirgiL. Cornell Univ., Ithaca, N. Y. 214 University Ave.

SokolNIKoff, Asst. Prof. I. S. Univ. of Wisconsin, Madison, Wis.

Solt, M. R. Instr., Univ. of New Hampshire, Durham, N. H.

Sommervilie, Prof. D. M. Y. Victoria Univ. Coll., Wellington, New Zealand.

SosNow, G. Y. Prudential Insurance Co., Newark, N. J. 635 High St.

Spalding, Dean Julia. Christian Coll., Columbia, Mo.

SPARKS, Prof. F. W. Texas Technological Coll., Lubbock, Tex.

Sparrow, Prof. C. M. Univ. of Virginia, University, Va. West Lawn.

SPEAR, Prof. JosEPH. Northeastern Univ., Boston, Mass.

SPEEkER, Asso. Prof. G. G. Michigan State Coll., East Lansing, Mich. Box 861.

SPEnCElex, Asso. Prof. G. W. Miami Univ., Oxford, Ohio. 100 E. Walnut St.

SPEnCER, Prof. Mary C. Newcomb Coll., New Orleans, La.

SPERLING, A. A. Instr., Univ. of Wisconsin, Milwaukee Branch, Milwaukee, Wis. 15854 th St.

SpErry, MaY J. Instr., Syracuse Univ., Syracuse, N. Y. Math. Dept., Coll. of Liberal Arts.

Sperry, Asst. Prof. Pauline. Univ. of California, Berkeley, Calif. 1194 Cragmont Ave.

Spicer, Prof. C. A. Western Maryland Coll., Westminster, Md.

SPINKS, M. J. Engineer, Champion Bridge Co., Wilmington, Ohio.

Spooner, Prof. C. C. Northern State Teachers Coll., Marquette, Mich. 210 E. Prospect St.

Sprague, Asso. Prof. A. H. Amherst Coll., Amherst, Mass. 17 College St.

SRIVASTAVA, P. L. Univ. of Allahabad, India.

StAFFord, AnNa A. St. John Baptist School, Mendham, N. J.

StAFFord, Asst. Prof. Elizabetr T. Texas Technological Coll, Lubbock, Tex. 2309 10th St.

Stager, Prof H. W. Salinas Junior Coll., Salinas, Calif. Box 871.

Stanley, H. S. Lehigh Univ., Bethlehem, Pa.

Staniland, A. E. Instr., Univ. of Pittsburgh, Pittsburgh, Pa. 107 Alumni Hall.

Stanwick, C. A. Electrical Engineer. 33 Beech St., East Orange, N. J.

STARK, Dr. F. R. Western Union Telegraph Co, 195 Broadway, New York, N. Y.

Stark, Asst. Prof. Marion E. Wellesley Coll., Wellesley, Mass.

Starke, Asso. Prof. E. P. Rutgers Univ., New Brunswick, N. J.

Starr, Asst. Prof. Elva E. Box 535, Alfred, N. Y.

SteCk, C. C. Spaulding Fibre Co., Tonawanda, N. Y. 15 Covington Road, Buffalo, N. $Y$.

Stecker, Mrs. Ormelle H. Instr., Pennsylvania State Coll., State College, Pa. Highland Ave. and Miles St.

Steed, Asst. Prof. D. V. Univ. of Southern California, Los Angeles, Calif.

StEgGall, Prof. J. E. A. University Coll., Dundee, Scotland.

StemLey, Dr. L. L. Associate, Univ. of Illinois, Urbana, Ill. Box 156, University Station.

SteinerT, E. E. Instr., Union Coll., Schenectady, N. Y. 9 Becker St. 
Steinmetz, Mary E. 545 Centre Ave., Reading, $\mathrm{Pa}$.

StepheNs, Dean R. P. Univ. of Georgia, Athens, Ga.

†Stetson, Prof. I. M. Coll. of William and Mary, Williamsburg, Va.

Stevenson, Asst. Prof. A. F. C. Univ. of Toronto, Toronto, Canada. Math. Dept. Stevenson, Asso. Prof. Guy. Univ. of Louisville, Louisville, Ky.

STEWART, L. B. Univ. of Toronto, Toronto, Canada.

STEWART, R. M. Dominion Observatory, Ottawa, Canada.

\$STILL WELL, Prof. S. E. Western and Southern Life Insurance Co., 4th and Broadway, Cincinnati, Ohio.

Stocker, Asso. Prof. J. E. Lehigh Univ., Bethlehem, Pa. 537 Center St.

Stokes, Ellen C. State Teachers Coll., Albany, N. Y.

Stone, Asst. Prof. M. H. Harvard Univ., Cambridge, Mass. 39 Walker St.

§STONE, MorRIs. Power Engineerng Dept, Westinghouse Electric Co., E. Pittsburgh, $\mathrm{Pa}$.

StOnE, Prof. ORMOND. Director of The Leander McCormick Observatory, Univ. of Virginia, University, Va. Clifton Station, Va.

Stone, Asso. Prof. R. B. Purdue Univ., Lafayette, Ind. 615 Russell St., W. Lafayette.

Stouffer, Prof. E. B. Univ. of Kansas, Lawrence, Kans. 1019 Maine St.

Stoughton, Leil A R. 270 Everit St., New Haven, Conn.

Stowell, Prof. C. J. McKendree Coll, Lebanon, Ill. Box 137.

Strom, Prof. C. W. Luther Coll., Decorah, Ia.

Strong, Dr W. M. Asso. Actuary, Mutual Life Insurance Co., 34 Nassau St., New York, N. Y.

STruik, Asso. Prof. D. J. Massachusetts Inst. of Technology, Cambridge, Mass.

Sturdivant, Dr. J. H. California Inst. of Technology, Pasadena, Calif. Gates Chemical Laboratory.

Suesman, W. P. Secretary, John M. Dean Co., 241 Weybosset St., Providence, R. I. 237 Oaklawn Ave., Meshanticut, $R$. $I$.

Sufra, Prof. Mary C. Elmira Coll., Elmira, N. Y.

Sullivan, Asst. Prof. C. T. McGill Univ., Montreal, Canada. Engineering Bldg. SutTon, Dr. Flora D. 1735 Bolton St., Baltimore, Md.

\$SwaIn, Prof. G. F. Harvard Univ., Cambridge, Mass. Pierce Hall, Oxford St.

Swartzel, Prof. K. D. Univ. of Pittsburgh, Pittsburgh, Pa. 4360 Center Ave.

§Swenson, OSCAR. Pacific Mutual Life Insurance Co., Los Angeles, Calif.

†Swirt, Prof. ElijaH. Univ, of Vermont, Burlington, Vt. $415 \mathrm{~S}$. Willard St.

SwINFord, Dr. L. H. Instr., Univ. of California, Berkeley, Calif.

SwINGLE, Dr. P. M. Instr., Ohio State Univ., Columbus, Ohio. 8 University Place.

Synge, Prof J. L. Trinity Coll., Dublin, Ireland.

Szabo, PaUl, JR. 921 Trinity Ave., Bronx, N. Y.

Szasz, Prof. OTto. Univ. of Frankfurt, Frankfurt a.M., Germany. Kettenhofweg $99 I$.

fTaber, Prof. Henry. Clark Univ., Worcester, Mass. 2 Pleasant Place.

TABER, T. S. Instr. Kern County Junior Coll., Bakersfield, Calif.

Takasu, Asst. Prof. Tsurusaburo. Tôhoku Imperial Univ., Sendai, Japan.

Tamarkin, Prof. J. D. Brown Univ., Providence, R. I.

TAN, Prof. V. A. Univ. of the Philippines, Manila, P. I.

TTANNER, Prof. J. H. Cornell Univ., Ithaca, N. Y.

Tanzola, J. J. Asst. Actuary Auditor, Pension Division, Dept. of Finance, New York, N. Y. 2041 Watson Ave., Bronx.

Tappan, Prof. A. Helen. Western Coll. for Women, Oxford, Ohio.

§TARBELL, T. F. Actuary, Travelers Insurance Co., 700 Main St., Hartford, Conn.

TARTler, Alexander. Instr., Drexel Inst., 32d and Chestnut Sts., Philadelphia, Pa.

Tate, Asst. Prof. Herbert. McGill Univ., Montreal, Canada.

TAte, Prof. Jennie L. McMurry Coll,, Abilene, Tex. 1301 Orange St.

Tavani, Francesco. 92 Loughborough Road, London, S. W. 9, England.

TAYLOR, Prof. Eugene. Univ. of Idaho, Moscow, Id.

TAYLOR, Prof. E. H. Eastern State Teachers Coll., Charleston, Ill. 885 7th St.

§TAYLOR, H. D. Research Dept., General Electric Co., Schenectady, N. Y.

TAYLOR, Prof. J. H. George Washington Univ., Washington, D. C.

TAYloR, Asst. Prof. J. S. Univ. of Pittsburgh, Pittsburgh, Pa. 103 Alumni Hall.

TAYLOR, Prof MLdREd E. Mary Baldwin Coll., Staunton, Va.

TeACH, Asst. Prof. V. B. Armour In'st. of Technology, Chicago, Ill. 6250 Harper Ave. 
§Terry, I. A Electrical Engineer, A C. Engineering Dept, General Electric Co, Schenectady, N Y.

Theobald, Prof. John. Columbia Coll., Dubuque, Ia. Loras Hall.

Thielman, H. P. Ohio State Univ., Columbus, Ohio

\$ThiesmeYer, Mitdpred G. Hunter Coll. High School, New York, N. Y. 81 Anderson Ave., Scarsdale, N. Y.

Thressen, H. B. Brown Univ., Providence, R. I.

Thomas, Prof. C. F. Case School of Applied Science, Cleveland, Ohio.

Thomas, Prof. Evan. Univ. of Vermont, Burlington, Vt. Coll. of Engineering.

§Thomas, E. E Pacific Mutual Life Insurance Co., Los Angeles, Calif.

\$Thomas, E. M. John Hancock Mutual Life Insurance Co., 197 Clarendon St., Boston, Mass.

Thomas, Asst. Prof. J. M. Univ. of Pennsylvania, Philadelphia, Pa. Bennett Hall.

Thomas, Asst. Prof T. Y Princeton Univ., Princeton, N. J. 42 Harethorne Ave.

Thompson, Asst. Prof. E L Texas Technological Coll., Lubbock, Tex

Thompson, Dr Helen. Yale Univ., New Haven, Conn. 52 Hillhouse Ave.

Trompson, J. E. Instr, Pratt Inst., Brooklyn, N. Y. $510 \mathrm{~W}$ 113th St., New York, N. $Y$.

Thompson, J. S. Mutual Benefit Life Insurance Co., Newark, N. J.

Thompson, Dr. L T. E. Physicist, Naval Proving Ground, Dahlgren, Va.

Thompson, Dr. W. R. Yale Univ., New Haven, Conn. $186 \mathrm{Elm}$ St.

Thomson, Dr. Euritu. Consulting Engineer. Director, Thomson Research Laboratory, 22 Monument Ave., Swampscott, Mass.

Thorndyke, Prof E. L. Teachers Coll., Columbia Univ., New York, N. Y

Thorne, H. H. The Fisher Library, Univ. of Sydney, Sydney, N. S. Wales, Australia.

Thorne, Asst. Prof. P. L. New York Univ., University Heights, New York, N. Y.

Thornton, Prof. R. A. Talladega Coll , Talladega, Ala.

ThuRow, W. H. Mullerstr. 30, Berlin N. 65, Germany.

ThuRston, H. S. Instr, Univ. of Alabama, University, Ala.

Tienzo, Asst. Prof. Telesforo. Univ. of the Philippines, Manila, P. I

Tillson, B. F. Consulting Engineer, Mountain Ave., N. Caldwell, N. J.

Tilly, Asst. Prof. Arthur. New York Univ., New York, N. Y.

\$Timoshenko, Prof. Stepan. University of Michigan, Ann Arbor, Mich

Tinner, Prof. J. C. Bishop Coll., Marshall, Tex.

Tolman, Prof. R. C. California Inst. of Technology, Pasadena, Calif.

Toops, Prof H. A. Ohio State Univ, Columbus, Ohio.

Torrance, C. C. Instr, Cornell Univ., Ithaca, N. Y. 614 Wyckoff Road,

Torrey, Asst Prof. Marian M. Goucher Coll., Baltimore, Md.

Touton, Vice-Pres. F. C. Univ. of Southern California, Los Angeles, Calif

TownSENd, Prof. E. J. Univ of Illinois, Urbana, Ill. $510 \mathrm{John}$ St., Champaign, Ill.

Tracey, Asso. Prof. J. I. Yale Univ., New Haven, Conn. 84 McKınley Ave.

TrEvor, Prof. J. E. Cornell Univ, Ithaca, N. Y. Rockefeller Hall.

TRIPP, Prof. M. O. Wittenberg Coll., Springfield, Ohio. $218 \mathrm{~W} . \mathrm{Cecul}$.

TrJitzinsky, Dr. W. J. National Research Fellow. Brown Univ, Providence, R. I.

§TRUMP, P. L. Instr., Univ. of Wisconsin, Madison, Wis. 405 North Hall.

TRYon, G. M. V. Fenton, Mich.

§TUlLER, ANNITA. 365 E. 2d St., Brooklyn, N. Y.

Turner, Asso. Prof. Bird M. Univ. of West Virginia, Morgantown, W. Va. 107 High St

Turner, Asso. Prof. J. S. Iowa State Coll., Ames, Ia. 2514 Knapp St.

\$TURRITTIN, H. L. Instr, Univ. of Wisconsin, Madison, Wis.

§TUTrLe, A. M. Instr., Univ. of Wisconsin, Madison, Wis.

Tyler, Prof. H.W. Massachusetts Inst. of 'Technology, Cambridge, Mass. General Secretary, A. A. U. P. 26 Jackson Place, Washingion, D. C.

Udinski, Asst. Prof. W. P. 419 S. Alamo St., San Antonio, Tex.

Uhler, Asso. Prof. H. S. Yale Univ, New Haven, Conn. 108 Thornton St., Hamden, Conn.

ULRICH, F. E. Instr., Union Coll., Schenectady, N. Y. 37 Ray St.

Underaill, Asso. Prof. A L. Univ. of Minnesota, Minneapolis, Minn. 100 Folwell Hall.

Unseld, G. P. West High School, Salt Lake City, Utah. 1359 Glenmore St

Upton, Prof. C. B. Teachers Coll, Columbia Univ., New York, N. Y.

URNER, Dr. S. E. 1725 18th St., Bakersfield, Calif.

Uspensky, Prof. J. V. Stanford Univ., Stanford University, Calif. 
Vallarta, Asst. Prof. M. S. Massachusetts Inst. of Technology, Cambridge, Mass. SVan ArNam, R. N. Lehigh Univ., Bethlehem, Pa.

VAN Buskirk, Prof. H. C. California Inst. of Technology, Pasadena, Calif.

\$VANDERslice, J. L. Princeton Univ., Princeton, N. J.

VAN DER VRIES, Dr. J. N. Manager, North Central Division, Chamber of Commerce of the United States, Chicago, Ill. 38 S. Dearborn St.

Vandiver, Asso. Prof. H. S. Univ. of Texas, Austin, Tex. Care of Guaranty Trust Co. of New York, 4 Place de la Concorde, Paris, France.

†VAN ORSTRAND, Dr. C. E. Lecturer, George Washington Univ., Washington, D. C. 1607 31st St., N.W.

VAN Velzer, Prof.'C. A. Carthage Coll., Carthage, Ill. 903 Buchanan St.

VAN VleCk, Prof. E. B. Univ. of Wisconsin, Madison, Wis. $519 \mathrm{~N}$. Prnckney St.

VASS, J. I. Instr, Univ. of Wisconsin, Extension Division, Milwaukee, Wis. 619 State St.

Vatnsdal, J. R. Instr, State Coll. of Washington, Pullman, Wash. 702 Campus Ave.

Vearch, Asst. Prof. R. W. Univ. of Tulsa, Tulsa, Okla.

tVeblen, Prof. Oswald. Princeton Univ., Princeton, N. J.

Vedder, Asst. Prof. J. N. Union Coll, Schenectady, N. Y.

Vedova, Asst. Prof. G. C. St. John's Coll., Annapolis, Md.

Verse, Asst. Prof. C. H. Univ. of West Virginia, Morgantown, W Va.

Vernon, E. L. Instr., Univ. of Wisconsin, Madison, Wis. Chemistry Bldg.

Vexler, Leonard. Technology Club, 38th St. and Madison Ave, New York, N. Y.

\$VICKERX, C. W. Instr., Univ. of Texas, Austin, Tex.

VirTs, R. O. Central High School, Fort Wayne, Ind.

Vivian, Dean Roxana H Hartwick Coll., Oneonta, N. Y.

Volterra, Prof. VITo. Üniv. of Rome, Rome, Italy. Via in Lucina 17.

Waddell, Mary E. G. Univ. of Toronto, Toronto, Canada. 72 Madrson Ave.

\$WAGNER, C. C. Univ. of Michigan, Ann Arbor, Mich. $333 \mathrm{E}$. Willam St.

\$WAGNER, E. H. Univ. of Michigan, Ann Arbor, Mich. 1709 Jackson Ave.

WAgner, Prof. P. S. Lebanon Valley Coll., Annville, Pa. 343 E. Main St.

Wagner, W. J. Instr., Allegheny Coll., Meadville, Pa. Cochran Hall.

WAfLERT, H. E. Instr., Washington Square Coll., New York Unıv, New York, N. Y. 61 Oakview Ave., L. I., N. Y.

Wartrn, Prof. G. E. Univ. of Missouri, Columbia, Mo. 1401 Anthony St.

Walker, Asso. Prof. Evelyn. Hunter Coll., Park Ave. and 68th St., New York,

N. Y. 287 N. Ocean Ave., Patchogue, Long Island, N. Y.

Walker, Rev. G. W. 47 Grove St., Perry, N. Y.

Walker, R. J. 131 Cedar Blvd, Mt Lebanon, Pittsburgh, Pa

WaLL, Asst. Prof. H. S. Northwestern Univ., Evanston, Ill.

WALSH, C. M. Bellport, L. I., N. Y.

WaLSH, Asso. Prof J. L. Harvard Univ., Cambridge, Mass. 547 Widener Library.

WaLter, R. M. Instr., New Jersey Coll.' for Women, New Brunswick, N. J.

Walton, Asst Prof. T. O. Kalamazoo Coll., Kalamazoo, Mich.

WArd, Asst. Prof. L. E. Univ. of Iowa, Iowa City, Ia. 127 Physics Bldg.

Ward, Asst. Prof. Morgan. California Inst. of Technology, Pasadena, Calif. \$Warner, Prof E. P. Massachusetts Inst. of Technology, Cambridge, Mass.

WARREN, Prof. L. A. H. Univ. of Manitoba, Winnipeg, Canada. 64 Niagara St.

Washburne, A. C. Actuary, Berkshire Life Insurance Co., Pittsfield, Mass.

WATKEYS, Prof. C. W. Univ. of Rochester, Rochester, N. Y.

Watson, Prof. G. N. Univ. of Birmingham, Edmund St., Birmingham, England. WatT, W. A. Pilot Life Insurance Co., Greensboro, N. C.

Watts, Virginia. 318 Walnut St., Bellevue, Ky.

Wear, Asso. Prof. L. E. California Inst. of Technology, Pasadena, Calif.

Weatherburn, Prof. C E Univ of Western Australia, Perth, W Australia.

Weaver, Prof.' J. H. Ohio State Univ., Columbus, Ohio. Hilliard, Ohio.

Weaver, Prof. Warres. Univ. of Wisconsin, Madison, Wis. 306 North Hall. WEBB, H. E. 12 Irving Place, Summit, N. J.

§WeBB, Louise. Univ. of Wisconsin, Madison, Wis. 252 Langdon St.

WebB, R. M. Kansas City Life Insurance Co., Kansas City, Mo. Box 1174.

Webber, Asso. Prof. W. J. Univ. of Toronto, Toronto, Canada.

Webber, Prof. W. P. Louisiana State Univ., Baton Rouge, La.

Weber, Prof. L. R. Friends Univ., Wichita, Kans.

Weber, Dean W. W, Lander Coli., Greenwood, S. C. 321 Stanley Ave. 
Webster, Prof. D. L. Stanford Univ., Stanford University, Calif. Box 52.

\$WEBSTER, Hosea. Babcock and Wilcox Co., 85 Liberty St. New York, N. Y. WeChSler, A. L. Instr., Columbia Univ., New York, N. Y. 27 W. $72 d$ St., Apt 712.

tWedderburn, Prof. J. H. M. Princeton Univ., Princeton, N. J. Box 53.

WeEks, Prof. Dorothy W. Wilson Coll , Chambersburg, Pa

\$WEgNer, K. W. Univ, of Wisconsin, Madison, Wis. North Hall.

WeIDA, Asso. Prof. F. M. George Washington Univ., Washington, D. C.

Weinstein, Clement. Instr. Univ. of Pennsylvania, Philadelphia, $\mathrm{Pa}$.

WeIsNer, Asst. Prof. LouIs. Instr., Hunter Coll., Park Ave. and 68th St., New York, N. Y.

Weiss, Asst. Prof. Marie J. H. Sophie Newcomb Memorial Coll., New Orleans, La.

WeLCH, Pres. A. A. Phoenix Mutual Life Insurance Co., Hartford, Conn. $79 \mathrm{Elm}$ $S t$.

Wells, Asst. Prof. E. H. Univ. of New Hampshire, Durham, N. H.

Wells, Prof. Mary E. Vassar Coll., Poughkeepsie, N. Y.

Wells, N. W. Chemist, The Texas Co, Port Arthur, Tex. 523 Stillwell Blvd.

Wells, Prof. R. A. Park Coll., Parkville, Mo.

Wells, Asst. Prof. V. H. Williams Coll., Williamstown, Mass. 3 Chapin Court.

WernICKe, Dr. Paul. U. S. Patent Office, Washington, D. C. 3600 South Dakota Ave., N.E.

West, Prof. T. P. Univ. of Idaho, Southern Branch, Pocatello, Id. 944 E. Carter St.

Wester, Prof. C. W. State Teachers Coll., Cedar Falls, Ia. 2312 Olvve St.

†Western, Dr. A. E. 47 Lansdowne Road, London, W. 11, England.

Westrall, Prof. W. D. A. Univ. of Missouri, Columbia, Mo. 11 S. Glenwood Ave.

Westlund, Prof. Jacoв. Purdue Univ., Lafayette, Ind. 1820 Scott St.

\$WeTHERILL, LynN. Engineer, General Electric Co., Pittsfield, Mass. 10 Superior $S t$.

Wetzel, R. A. Townsend Harris Hall, Coll. of the City of New York, New York, N. Y. 218 Tecumseh Ave., Mt. Vernon, N. Y.

WeXler, Chardes. Instr., Harvard Univ., Cambridge, Mass. 25 Irving Terrace.

Weyl, Prof. Hermann. University of Göttingen. Merkelstrasse 3, Gottingen, Germany.

WheELer, A. H. North High School, Worcester, Mass. 16 Bellevue St.

Wheeler, Dr. Anna Pell (Mrs.). Lecturer, Bryn Mawr Coll., Bryn Mawr, Pa. 109 Broadmead, Princeton, N.J.

Wheeler, Asst. Prof. J. J. Univ. of Kansas, Lawrence, Kans.

\$WheEler, R. A. Pacific Mutual Life Insurance Co., Los Angeles, Calif.

WHELAN, Dr. Rose A. Instr, Univ. of Rochester, Rochester, N. Y. 91 N. Leyden St., Campello, Mass.

White, Asst. Prof. G. W. Coll. of the Pacific, Stockton, Calif.

White, Prof. H. S. Vassar Coll., Poughkeepsie, N. Y.

White, Asst. Prof. Marion B. Carleton Coll., Northfield, Minn.

Whited, Dr. Wulis. State Dept. of Highways, Harrisburg, Pa. 26 S. 3d St.

Whitemead, H. E. C. Commonwealth Fellow. Princeton Univ., Princeton, N. J.

WhITford, D. E. Instr., Polytechnic Inst., Brooklyn, N. Y. 34 Ames St., Phenix, $R$. I.

Whitrord, Asso. Prof. E. E. Coll. of the City of New York, New York, N. Y. 535 W. 110th St.

Whiting, MABel G. Santa Ana Junior Coll., Santa Ana, Calif. 506 E. Chestnut Ave.

Whitman, Asst. Prof. E. A. Carnegie Inst. of Technology, Pittsburgh, Pa.

Whitney, A. W. National Bureau of Casualty and Surety Underwriters, 1 Park Ave, New York, N. Y. Room 907.

WhitNey, Hassler. Instr., Yale Univ., New Haven, Conn. 188 Bishop St.

tWhitraker, Prof. E. T. Univ, of Edinburgh, Edinburgh, Scotland. 48 George Square.

†Whittemore, Asso. Prof. J. K. Yale Univ, New Haven, Conn. 45 Lincoln St.

Whyburn, Asst. Prof. G. T. Johns Hopkins Univ., Baltimore, Md.

WhybURN, Asso. Prof. W. M. Univ. of California at Los Angeles, Los Angeles, Calif.

Wible, Prof. W. M. Intermountain Union Coll., Helena, Mont. 1735 Winne Ave.

WICK, R. F. Electrical Engineer. 215 W. 23d St., New York, N Y.

Widder, Prof. D. V. Bryn Mawr Coll., Bryn Mawr, Pa.

WIDMARK, L. E. Star Electric Motor Co., Newark, N. J.

Wiener, Asst. Prof. Norbert. Massachusetts Inst. of Technology, Cambridge, Mass, 
\$Wieseman, R. W. A. C. Engineering Dept., General Electric Co., Schenectady, N. Y.

Wiggin, Evelyn P. Stratham, N. H.

Wightman, E. C. Actuary. Care of Lincoln National Life Insurance Co., Fort Wayne, Ind.

WILcox, Asst. Prof. H. B. Univ. of Minnesota, Minneapolis, Minn.

WIICZEWSKI, Dr. JosEPH. St. Xavier Coll., Evanston Station, Cincinnati, Ohio. Wilczynski, Prof. E. J. Univ. of Chicago, Chicago, Ill.

Wilder, Asst. Prof. C. E. Dartmouth Coll., Hanover, N. H. 5 Dana Road. WIIDER, Asso. Prof. R. L. Univ. of Michigan, Ann Arbor, Mich. 1209 Packard St. WILEY, Prof. F. B. Denison Univ., Granville, Ohio.

Wulcox, Prof. W. F. Cornell Univ. Ithaca, N. Y.

Wultams, Dr. A. R. Instr., Univ, of California, Berkeley, Calif. 416 Y.M. C. A.

Wirliams, Dr. EmILY C. (Mrs. W. H.). 7 E. 63d St., New York, N. Y.

Willrams, Prof. F. B. Clark Univ., Worcester, Mass.

Williams, Prof. F. G. Susquehanna Univ., Selinsgrove, Pa.

Wuliams, Asst. Prof. F. H. M. Drexel Inst., Philadelphia, Pa. Room 240, $139 \mathrm{~W}$. Albanus St., Olney.

Wrlliams, Prof. H. B. Columbia Univ., New York, N. Y. 630 W. 168th St.

Wrliams, Dean J. E. Virginia Polytechnic Inst., Blacksburg, Va.

Williams, Prof. K. P. Indiana Univ., Bloomington, Ind. 533 E. $3 d$ St.

Wrrrams, Asso. Prof. W. L. G. McGill Univ., Montreal, Canada.

Willamson, Prof. C. O. Coll. of Wooster, Wooster, Ohio. 1141 Beall Ave.

Whitramson, Dr. JoHs. Associate, Johns Hopkins Úniv., Baltimore, Md.

Willis, E. J. Consulting Engineer. Box 416, Richmond, Va.

WrLlis, RUBY. Walnut High School, Natick, Mass.

WILlIs, W. J. Patent Attorney, Union Carbide and Carbon Research Laboratories, Inc., New York, N. Y. 248-24 89th Ave., Bellerose, L. I., N. Y.

WILMER, F. L. Odebolt, Ia.

Wnson, Asso. Prof. A. H. Haverford Coll., Haverford, Pa.

Wilson, C. R. Instr., Rutgers Univ., New Brunswick, N. J.

WIIson, Prof. E. B. Harvard Univ., Cambridge, Mass. 55 Van Dyke St., Boston, 17, Mass.

Wilson, Elizabeth W. Actuary. Mather Courts, Apt. 42, Cambridge, 38, Mass.

Wrison, Prof. H. A. Rice Inst., Houston, Tex.

Wuson, Asso. Prof. L. T. U.S. Naval Academy, Annapolis, Md. 20 Thompson St.

Wilson, Prof. N. R. Univ, of Manitoba, Winn peg, Canada. 989 Grosvenor Ave.

Wirson, Prof. W. A. Yale Univ., New Haven, Conn. 1960 Chapel St.

Wrison, Dean W. H. Univ. of Florida, Gainesville, Fla.

†WILton, Prof. J. R. Univ. of Adelaide, Adelaide, South Australia.

Winger, Prof. R. M. Univ. of Washington, Seattle, Wash.

Winston, JEAn. Instr., Univ. of Cincinnati, Cincinnati, Ohio.

Winters, F. W. Bell Telephone Laboratories, 463 West St., New York, N. Y.

Wirte, H. P. Instr., Coll. of the City of New York, New York, N. Y.

Wistand, G. W. 5134 Carthage Ave., Norwood, Ohio.

Wirmer, Asst. Prof. E. E. Randall Morgan Laboratory, Univ. of Pennsylvania, Philadelphia, Pa. Physics Dept.

Wolfe, Asst. Prof. C. L. E. California Inst. of Technology, Pasadena, Caifl.

WolfE, Asso. Prof. H. E. Indiana Univ., Bloomington, Ind. 314 N. Washington St.

Wolfenden, H. H. Consulting Actuary. 182 Rosedale Heights Drive, Toronto, 5, Ont., Canada.

Wolff, Prof. H. C. Drexel Inst., Philadelphia, Pa.

Wong, Asst Prof. B. C. Univ, of California, Berkeley, Calif. 1933 Grant St.

WoNG, Y. K. Univ, of Chicago, Chicago, Ill. 5629 Dorchester Ave.

Woo, Kamcheung. Univ. of California, Berkeley, Calif. 2407 Fulton St.

§Wod, Douglas. Missourı State Life Insurance Co., St. Louis, Mo.

Wood, Dean Frederick. Hamline Univ., St. Paul, Minn.

Wood, Asst. Prof. F. E. Northwestern Univ., Evanston, Ill.

Wood, Rose B Greenville Woman s Coll., Greenville, S. C. 121 8th St., N. E., Allanta, Ga.

Wood, Prof. Ruth G. Smith Coll., Northampton, Mass. 249 Crescent St.

tWood, Wal Ter. Engineer. 400 Chestnut St., Philadelphia, Pa.

WoodarD, Prof. D. W. Howard Univ., Washington, D. C. 127 W St., N. W.

Woods, Prof. F. S. Massachusetts Inst. of Technology, Cambridge, Mass.

Woods, Prof. L. P. Northeastern State Teachers Coll., Tahlequah, Okla. 529 Seminary Ave. 
Woods, Asst. Prof, Roscoe. Univ. of Iowa, Iowa City, Ia. 221 Physics Bldg. Woolard, Asst. Prof. E. W. George Washington Univ., Washington, D. C.

Woolf, S. H. Photographer. Chicago Beach Hotel, Chicago, III.

WORTH, C. R. Instr., Univ, of Arkansas, Fayetteville, Ark.

Worthington, Asst. Prof. Euphemia R. Univ. of California at Los Angeles, Los Angeles, Calif.

Wren, Dean F. G. Tufts Coll., Tufts College, Mass.

Wren, Asso. Prof. F. L. George Peabody Coll., Nashville, Tenn.

Wright, Asst. Prof. Frances M. Elmira Coll., Elmira, N. Y.

WUNDER, Dean C. N. Univ. of Mississippi, University, Miss.

Wyant, E. Kathryn. Instr., Univ. of Missouri, Columbia, Mo. 213 Engineering Bldg.

Wrute, Asst. Prof. C. C. Univ. of Iowa, Iowa City, Ia.

YaNAGrHara, K. Lecturer, Yamagata Higher School, Yamagata, Japan.

YANNEy, Prof. B. F. Coll. of Wooster, Wooster, Ohio. $666 \mathrm{~N}$. Bever St.

YaNosIK, G. A. New York Univ., University Heights, New York, N. Y. 52 Greenvale Ave.

Yates, W. H. Civil Engineer. $420 \mathrm{~W}$. 130th St., New York, N. Y.

Yeaton, Prof. C. H. Oberlin Coll., Oberlin, Ohio. 189 Forest St.

§YeruShalmy, JACOB. Instr., Johns Hopkins Univ., Baltimore, Md. 2126 Bolton St.

YoshIDA, Dr. Minow. 5th Sakuragoaka Shibuyacho, Tokyo, Japan.

Yost, Asst. Prof. D. M. California Inst. of Technology, Pasadena, Calif.

YounG, Asst. Prof. Jessica M. Washington Univ., St. Louis, Mo.

Young, Prof. J. W. Dartmouth Coll., Hanover, N. H.

Young, Asso. Prof. J. W. A. Univ. of Chicago, Chicago, Ill. 5422 Blackstone Ave.

Young, Asso. Prof. Mabel M. Wellesley Coll., Wellesley, Mass. 6 Norfolk Terrace.

Young, Margaret M. Instr., Hunter Coll., Park Ave. and 68th St., New York, N. Y. 1001 Anderson Ave.

Yowel., Asso. Prof. E. 1. Univ. of Cincinnati, Cincinnati, Ohio. 327 Griest Ave.

YUROW, Louis. 331 Woodward Bldg., Washington, D. C.

Zakarian, Zarmair. 615 East 15th St., New York, N. Y.

ZARISKI, Dr. OSCAR. Instr., Johns Hopkins Univ., Baltimore, Md. Box 1126.

Zehring, Asso. Prof. W. A. Purdue Univ., Lafayette, Ind. 303 Russell St., W. Lafayette.

Zeldin, Dr. S. D. Instr., Massachusetts Inst. of Technology, Cambridge, Mass. Zervos, Prof. Panaiotis. Univ. of Athens, Athens, Greece. Rue Sozopoleos 20. ZipP N, Leo. Instr., Pennsylvania State Coll., State College, Pa.

ZOBEL, Dr. O. J. American Telephone and Telegraph Co., 195 Broadway, New York, N. Y.

Number of Ordinary Members, October, 1928 (including 166 nominees of

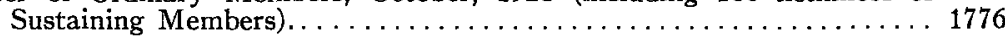

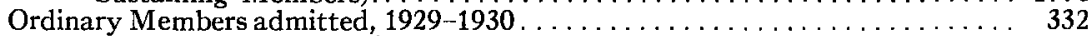

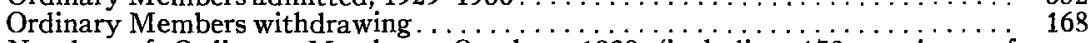

Number of Ordinary Members, October, 1930 (including 172 nominees of

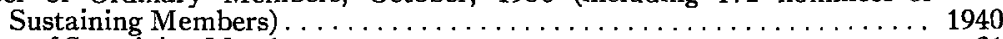

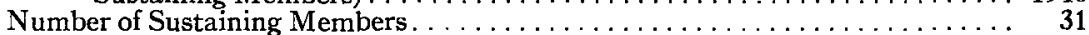

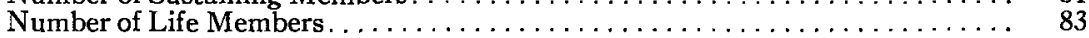

